\title{
WestVirginiaUniversity
}

THE RESEARCH REPOSITORY @ WVU

Graduate Theses, Dissertations, and Problem Reports

2011

\section{Drug-drug interactions: A machine learning approach}

William Wireko Mensah

West Virginia University

Follow this and additional works at: https://researchrepository.wvu.edu/etd

\section{Recommended Citation}

Mensah, William Wireko, "Drug-drug interactions: A machine learning approach" (2011). Graduate Theses, Dissertations, and Problem Reports. 4753.

https://researchrepository.wvu.edu/etd/4753

This Thesis is protected by copyright and/or related rights. It has been brought to you by the The Research Repository @ WVU with permission from the rights-holder(s). You are free to use this Thesis in any way that is permitted by the copyright and related rights legislation that applies to your use. For other uses you must obtain permission from the rights-holder(s) directly, unless additional rights are indicated by a Creative Commons license in the record and/ or on the work itself. This Thesis has been accepted for inclusion in WVU Graduate Theses, Dissertations, and Problem Reports collection by an authorized administrator of The Research Repository @ WVU. For more information, please contact researchrepository@mail.wvu.edu. 


\title{
DRUG-DRUG INTERACTIONS: A MACHINE LEARNING APPROACH
}

\author{
William Wireko Mensah \\ Thesis submitted to the \\ College of Engineering and Mineral Resources \\ at West Virginia University \\ in partial fulfillment of the requirements \\ for the degree of \\ Master of Science \\ in \\ Computer Science
}

Donald A. Adjeroh, Ph.D., Chair

Arun Ross, Ph.D.

Elaine Eschen, Ph.D.

Lane Department of Computer Science and Electrical Engineering

Morgantown, West Virginia

2011

Keywords: Drug-Drug Interactions, Machine Learning, PU-Learning, Adverse Drug Reactions Copyright 2011 William W. Mensah 


\section{ABSTRACT \\ DRUG-DRUG INTERACTIONS: A MACHINE LEARNING APPROACH \\ William W. Mensah}

Automatic detection of drug-drug interaction (DDI) is a difficult problem in pharmacosurveillance. Recent practice for in vitro and in vivo pharmacokinetic drug-drug interaction studies have been based on carefully selected drug characteristics such as their pharmacological effects, and on drug-target networks, in order to identify and comprehend anomalies in a drug's biochemical function upon co-administration.

In this work, we present a novel DDI prediction framework that combines several drugattribute similarity measures to construct a feature space from which we train three machine learning algorithms: Support Vector Machine (SVM), J48 Decision Tree and K-Nearest Neighbor (KNN) using a partially supervised classification algorithm called Positive Unlabeled Learning (PU-Learning) tailored specifically to suit our framework.

In summary, we extracted 1,300 U.S. Food and Drug Administration-approved pharmaceutical drugs and paired them to create 1,688,700 feature vectors. Out of 397 drug-pairs known to interact prior to our experiments, our system was able to correctly identify $80 \%$ of them and from the remaining 1,688,303 pairs for which no interaction had been determined, we were able to predict 181 potential DDIs with confidence levels greater than $97 \%$. The latter is a set of DDIs unrecognized by our source of ground truth at the time of study.

Evaluation of the effectiveness of our system involved querying the U.S. Food and Drug Administration's Adverse Effect Reporting System (AERS) database for cases involving drug-pairs used in this study. The results returned from the query listed incidents reported for a number of patients, some of whom had experienced severe adverse reactions leading to outcomes such as prolonged hospitalization, diminished medicinal effect of one or more drugs, and in some cases, death. 


\section{ACKNOWLEDGMENTS}

This thesis would not have been possible without the support and guidance from a number of individuals who have made significant contributions, whether directly or indirectly, towards the preparation and successful completion of this work.

First and foremost, my utmost gratitude to Dr. Donald A. Adjeroh, Associate Professor, Lane Department of Computer Science and Electrical Engineering (LCSEE), West Virginia University, who proposed the main idea behind this work. Dr. Adjeroh's dedication to this project served as a source of motivation that helped fuel collaboration amongst intelligent minds towards its completion. In addition, I would like to acknowledge the remaining committee members, specifically:

Dr. Arun Ross, Associate Professor and Assistant Director at the Center for Identification Technology Research (CITeR), LCSEE, West Virginia University for enlightening us with machine learning and pattern classification techniques that provided the engine to facilitate this work, without which the framework we present would be non-existent.

Dr. Elaine Eschen, Associate Professor, LCSEE, West Virginia University, for imparting her expertise on the analysis of algorithms on us, specifically with respect to runtimes and optimization techniques, and thereby enabling the completion of this work in due time.

Outside the committee yet equally worth recognizing is Dr. Zheng Wanhong, MD, Psychiatrist and Assistant Professor at the Chestnut Ridge Psychiatric Hospital in Morgantown, West Virginia, for his guidance and ensuring the credibility of our work in the medical field; and also Jimmy Stemple, Ed.D., Assistant Director of Institutional Research, West Virginia University, for his support as well.

Finally, I would like express appreciation to my spouse, Codi Mensah and immediate family members, especially my mother, Letitia Adjei-Botchway and brother, Richard Mensah, for their support and encouragement throughout the course of this work. And also to friends and colleagues who have influenced this research in one way or another.

Last but not the least, I would like to thank the almighty God, for with Him, I believe I can do all things. 


\section{TABLE OF CONTENTS}

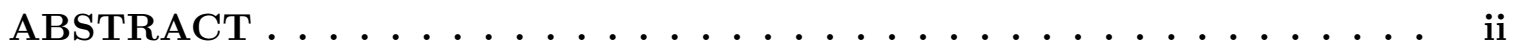

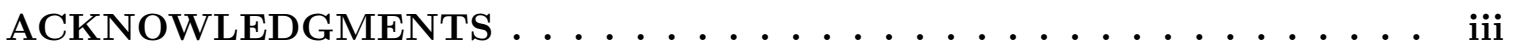

LIST OF TABLES $\ldots \ldots \ldots \ldots \ldots \ldots \ldots \ldots$ vii

LIST OF FIGURES $\ldots \ldots \ldots \ldots \ldots \ldots \ldots \ldots \ldots$ ix

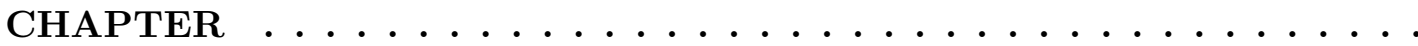

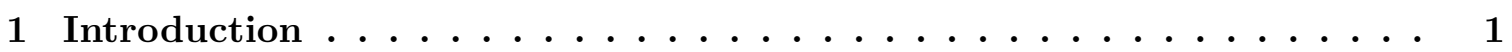

1.1 The Problem \& Motivation $\ldots \ldots \ldots \ldots \ldots \ldots$

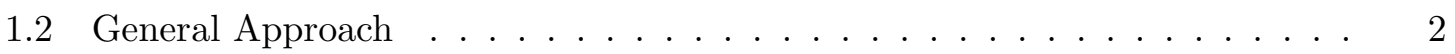

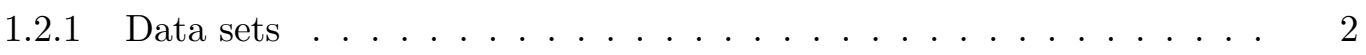

1.2.2 Drug Attributes \& Feature Space . . . . . . . . . . . . . 2

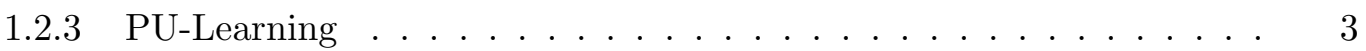

1.2.4 Performance Measurement . . . . . . . . . . . . . . . . 3

1.3 Thesis Contribution $\ldots \ldots \ldots \ldots \ldots \ldots \ldots \ldots \ldots$

1.4 Thesis Organization $\ldots \ldots \ldots \ldots \ldots \ldots \ldots \ldots \ldots$

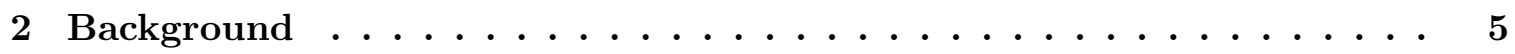

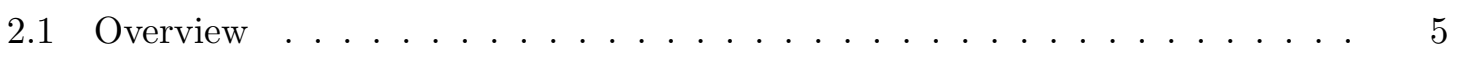

2.2 Related Work . . . . . . . . . . . . . . . . . . . 8

3 Methods ........................ 10

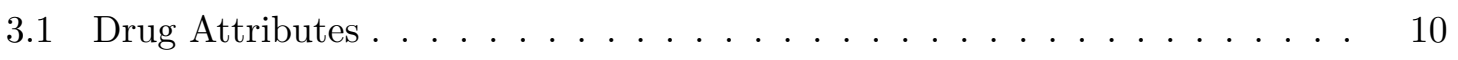

$3.1 .1 \quad$ Active Ingredient Similarity . . . . . . . . . . . . . . . . 11

3.1.2 Anatomical Therapeutic Similarity . . . . . . . . . . . . 12

3.1 .3 Protein-Protein Interaction $\ldots \ldots \ldots \ldots \ldots$ 
3.1.4 Protein Sequence Alignment . . . . . . . . . . . . . . . . 15

3.1.5 Chemical Compound Similarity . . . . . . . . . . . . . 16

3.2 Database Architecture . . . . . . . . . . . . . . . . 20

3.2 .1 Drugs Table . . . . . . . . . . . . . . . . . 20

3.2 .2 Active Ingredients Table . . . . . . . . . . . . . . . . 20

3.2.3 Protein Targets Table . . . . . . . . . . . . . . . . . . . . . . 20

$3.2 .4 \quad$ ATC Codes Table . . . . . . . . . . . . . . . . . . . . . . 21

3.2.5 Drugs-Active Ingredients Table . . . . . . . . . . . . . . . . . . 21

3.2.6 Drugs-Protein Targets Table . . . . . . . . . . . . . . . 21

3.2 .7 Protein-Protein Interaction Table . . . . . . . . . . . . . . 21

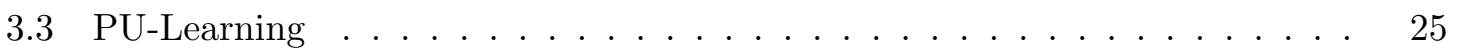

$3.3 .1 \quad \mathrm{EM} / \mathrm{S}-\mathrm{EM}$ Algorithm $\ldots \ldots \ldots \ldots \ldots . \ldots \ldots 27$

3.3 .2 Co-Training . . . . . . . . . . . . . . . . . 29

3.3.3 Tri-Training . . . . . . . . . . . . . . . . . . . . . . 29

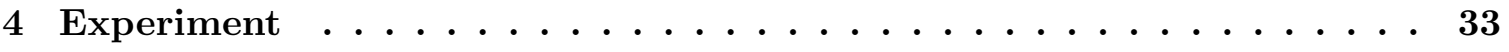

4.1 Experimental Setup . . . . . . . . . . . . . . . . 33

$4.1 .1 \quad$ Data Sets . . . . . . . . . . . . . . . . . 33

4.1 .2 Computational Environment . . . . . . . . . . . . . 34

4.1.3 Data Pre-processing . . . . . . . . . . . . . . . 34

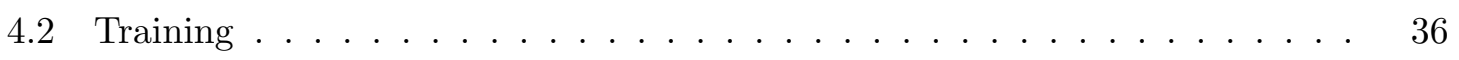

4.2 .1 Performance Measures . . . . . . . . . . . . . . 36

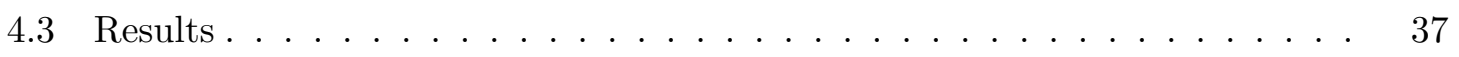

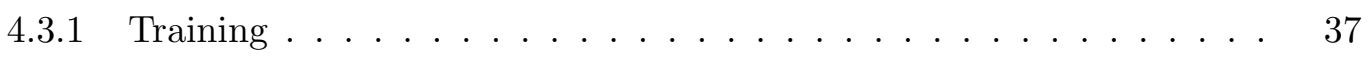

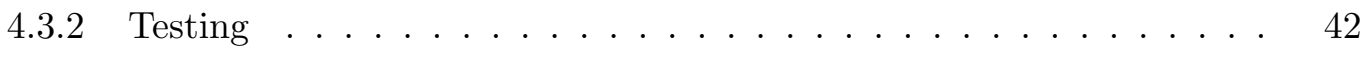

4.3 .3 Statistical Analysis . . . . . . . . . . . . . . . 45 
5 Conclusion and Future Work .................. 53

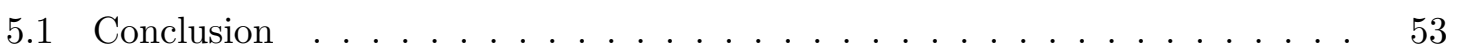

5.2 Future Work ............................. 54

5.3 Broader Application Domains . . . . . . . . . . . . . . 55

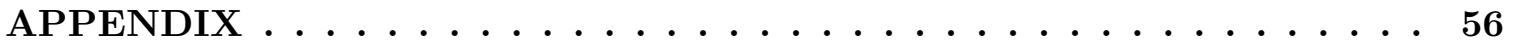

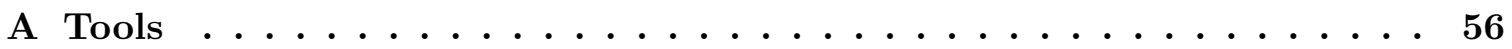

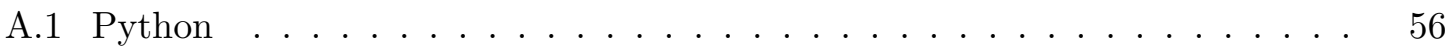

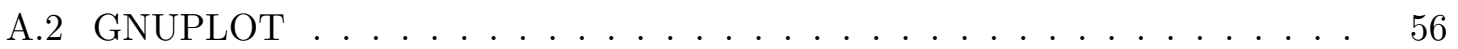

A.3 Cytoscape ........................... 56

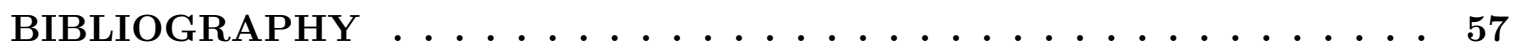




\section{LIST OF TABLES}

Table

Page

3.1 Anatomical Therapeutic Chemical classification for the anti-diabetic drug

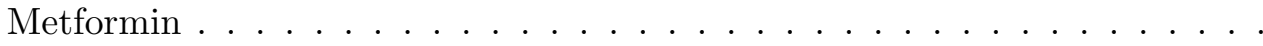

3.2 Attributes that constitute a feature vector in the dataset. Owing to the relatively small size of the positive set, drug-pairs comprising known DDIs are assigned IDs between 0 and 1000 while IDs for unlabeled pairs are greater than $1000 \ldots \ldots \ldots \ldots \ldots \ldots \ldots$

4.1 Top attributes selected during the feature-selection process. Each value is an average of 20 runs. . . . . . . . . . . . . . . . .

4.2 Potential Psychotropic Drug-Drug Interactions from 20 separate sets of medication lists for 20 patients. . . . . . . . . . . . . .

4.3 Potential Psychotropic DDI ADRs for drug-pairs listed in Table 4.2. The table also shows the most common side effects and number of cases reported in the 2010 Q4 AERS data set. . . . . . . . . . . . . . . . . 44

4.4 DDI for Psychotropic medications found in the unlabeled drug-pair dataset

4.5 Forty-one randomly selected attribute-values for attribute ppi_b (index 20). $\mathrm{K}, \mathrm{P}, \mathrm{U}$ represent known DDIs, predicted DDIs and unknown DDIs respectively. Columns containing no information (0.0) across all three rows have

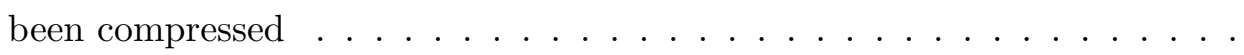


4.6 Twenty-one samples of data obtained from the process described in Table 4.5. The mean, standard deviation (std), minimum and maximum values for $\mathrm{K}$, $\mathrm{P}$ and $\mathrm{U}$ has been computed for each iteration. From the table, it can be observed that the $\mathrm{P}$ and $\mathrm{K}$ have similar distributions, both of which are different from U. . . . . . . . . . . . . . . . 


\section{LIST OF FIGURES}

Figure $\quad$ Page

3.1 Chemical structure similarity for FDA-approved drugs Abilify (KEGG ID: D01164) and Vistaril (KEGG ID: D00672). Longest Common Substring components have been marked with bold lines. . . . . . . . . . . . . . .

3.2 Generalized suffix tree for strings $S_{1}=x a b x a$ and $S_{2}=b a b x b a$. The first number at a leaf indicates the string; the second number indicates the starting position of the suffix in that string [1]. Edges marked with double lines highlight the path to the LCS, which is $a b x$ in this case. . . . . . . . . 18

3.3 Drug Database Architecture. The tables drugs_ar, drugs_atc, drugs_ai, drugs_pt, and ppi serve as bridges to the drugs, adverse_reactions, atc_codes, active ingredients and protein_targets tables. Although we deliberately omitted ADR information from our dataset, we later used it to prove the credibility of our system in Section $4.3 . \ldots \ldots \ldots \ldots \ldots \ldots$

3.4 Top 10 drugs from the drug database with the most active ingredients, most ATC classifications and most human protein targets. . . . . . . . . . 23

3.5 Distribution of drug-attributes stored in the database within which all drugs studied in this work are stored. . . . . . . . . . . . . . . . 24

3.6 PU-Learning procedure, showing incorporation of the spy technique with the EM Algorithm.. . . . . . . . . . . . . . . . . . . . . 28

4.1 Influence of the size of unlabeled data on PU Learning using all 21 attributes. The best output is realized when 700 unlabeled instances are used in training. 38

4.2 Impact of feature subset selection on 700 unlabeled instances. . . . . . . . . 41 ix 
4.3 Evaluation of SVM classifier on 100 positive and 100 unlabeled instances. .

4.4 Network showing drugs involved in known (blue nodes, 397 pairs) and predicted (red nodes, 181 pairs) drug-drug interactions. Blue edges indicate a connection between two nodes known to interact prior to experiments while red edges represent probable connections predicted by our system. . . . . .

4.5 F-Distribution showing critical value $\mathrm{F}(2,120)=3.07$, which is the starting point of the blue shaded region indicating the area within which the null

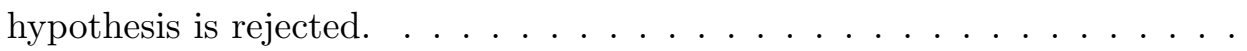

4.6 Distribution of Known, Predicted and Unlabeled instances based on mean values of attributes ppi_b (binary protein-protein interaction) and sfmsim (structural formula similarity) for 41 randomly selected drug-pairs, and repeated 100 times. Therefore each class (i.e. K, P and U) plotted consists of exactly 100 points, some of which overlap each other. The plot clearly shows a separation of clusters of $\mathrm{K}$ and $\mathrm{P}$ from $\mathrm{U} \ldots \ldots \ldots \ldots$ 


\section{Chapter 1}

\section{Introduction}

\subsection{The Problem \& Motivation}

Drug-drug interactions (DDIs) by nature tend to be extremely difficult to anticipate, costly to diagnose and treat correctly, and result in severe adverse drug reactions, some of which may be fatal. As new drugs emerge into the consumer market, it becomes crucial for both medical doctors and patients to understand the risk associated with co-administration of these drugs in order to anticipate any potential threats. Unfortunately, biological methods used today are quite cumbersome and relatively slow. Recently, a number of systems and publicly available databases have been created to facilitate the reporting of adverse drug events, but unfortunately, such databases only serve as sources of information and hence fail to identify specific drugs involved in adverse drug reactions. Current commercial software used in today's hospitals suffice to some extent when the drugs in question are limited to those whose adverse effects have already been reported; however, they are incompetent when prompted with new drugs or unusual drug combinations. For example, recent studies conducted by Vonbach et al [2] tested nine popular DDI screening software programs using profiles from hospital patients experiencing adverse drug effects attributed to DDIs. The outcome of their experiments showed that only $11 \%$ of the DDIs was found by all the programs on average. Individually, none of the programs was able to detect over $50 \%$ of the 
DDIs. Intuitively, if we can predict potential DDIs for both new drugs and those already in the hands of consumers, we would be able to set different levels of precautions for clinical drugs administered to patients very quickly, thereby reducing the cost involved in diagnosing and treating adverse drug reactions and saving lives in the long run.

\subsection{General Approach}

We collected a list of clinically approved drugs from the U.S. Food and Drug Administration's (FDA) drugs database [3], retrieved information pertaining to specific drug attributes for each drug and computed a number of similarity measures amongst these properties for multiple drug-pairs to construct a dataset. From this dataset, we identified known DDIs, with which we employed machine learning techniques to learn three classifiers. We then determined the best classifier and saved its model for future prediction of potential DDIs.

\subsubsection{Data sets}

The dataset used in this work is constructed from the pairing of 1,300 unique clinically approved drugs obtained from the FDA's drug database marked up in Structured Product Labeling (SPL) format [3]. Known DDI information was obtained from two sources of ground truth: Stockley's Drug Interactions [4] and general online sources. Information regarding interaction between protein targets for these drugs was extracted from the DrugBank database [5].

\subsubsection{Drug Attributes \& Feature Space}

For each drug, we gathered a specific set of attributes including its chemical structure, active ingredients, adverse drug reactions (ADR), Anatomical Therapeutic Chemical (ATC) classification codes, human protein targets, and protein interactions. Each drug's attributes were combined with those of another drug during the pairing phase to create a feature vector. However, ADRs were deliberately excluded from the feature space to be used in the validation of our system. Combination algorithms employed to construct the feature 
vectors include similarity measures such as Tanimoto's Coefficient and Longest Common Substring.

\subsubsection{PU-Learning}

DDIs confirmed by general online sources and Stockley's Drug Interactions [4], our sources of ground truth, comprised the positive set of drug-pairs. Because it is difficult to guarantee that two drugs do not interact, the remaining drug-pairs for which we couldn't confirm any reported interactions comprised the unlabeled set of drug-pairs, rather than negative drugpairs. The lack of negative instances in the dataset makes the use of traditional machine learning approaches very difficult. As a result, we adopt an alternate paradigm called Positive-Unlabeled Learning which strives to extract a set of reliable negative instances from the unlabeled set and use that, combined with the positive set, for training.

\subsubsection{Performance Measurement}

Evaluation of each classifier's performance was accomplished using Precision, Recall and F-measure metrics. Precision evaluates the measure of exactness, that is, amongst all DDIs retrieved by the system, how many were true DDIs? On the other hand Recall measures the completeness of the returned results. In other words, from the set of DDIs already known, how many of the DDIs returned by the system are in the set? F-measure is a harmonic mean between Precision and Recall that can simply be thought of as an average between the two metrics.

\subsection{Thesis Contribution}

The originality of the proposed method lies in the formalization of a drug-drug interaction inference as a semi-supervised machine learning problem and the integration of specific drug characteristics including their chemical structure, Anatomical Therapeutic Chemical (ATC) classification and human protein targets into a pharmacological space.

Our major contribution to the study of DDIs is the unfolding of an unexplored approach 
to addressing the problem. We provide a novel framework for predicting DDIs by pairing carefully selected drug attributes in a feature space in order to identify distinct similarities and learn on the patterns that are spawned when a variety of clinical drugs are included in this space. The framework presented facilitates the study of different causes of DDIs simultaneously. Furthermore, we introduce the problem of inverse feature vectors that arises from the pairing of drugs since doing so produces two distinct feature vectors that may be classified differently even though both feature vectors involve the same drugs. To circumvent this problem, we present a solution that could be applied even in the case where three or more drugs are involved in the construction of a single feature vector.

\subsection{Thesis Organization}

The remainder of this thesis is organized as follows: Chapter 2 gives an account of background and previous work that has been done in this area. The methods and data used in our experiment are discussed in Chapter 3. The actual experiment and results obtained are explained and analyzed in Chapter 4. Finally, Chapter 5 concludes the thesis, and in addition, outlines possible future work. Information regarding all software used in this work including their version numbers can be found in Appendix A. 


\section{Chapter 2}

\section{Background}

\subsection{Overview}

The Food, Drug \& Cosmetic (FD\&C) Act defines drugs, by their intended use, as "articles intended for use in the diagnosis, cure, mitigation, treatment, or prevention of disease" and "articles (other than food) intended to affect the structure or any function of the body of man or other animals" [6]. However, within the context of this work, we restrict the definition of a drug to clinically approved substances used to exert a pharmacological effect on the human body. For a drug to be effective, it must be exposed to the internal organs it is intended to act on. Generally, multiple drugs acting on the same site tend to interfere with each other so that the effect of one drug is either suppressed or magnified by the other. While some drug interactions may be beneficial (for example, the combination of amoxicillin and clavulanic acid), others can lead to severe side effects that are clinically harmful. The harmful effect of a drug interaction is often referred to as an Adverse Drug Reaction (ADR), technically defined as "a response which is noxious and unintended, and which occurs at doses normally used in man for the prophylaxis, diagnosis, or therapy of diseases, or for the modification of physiological function" [7]. The cost associated with drug-related problems in the United States from 1995 to 2000 increased from $\$ 76.6$ billion to $\$ 177.4$ billion $[8,9]$. Severity of such ADRs has resulted in early termination of development, refusal of approval, 
severe prescribing restrictions, and in some cases withdrawal of drugs from the market [10]. Recently, pharmacogenomic studies have led to the discovery of a connection between ADRs and DDIs indicating that the sharing of metabolic pathways by multiple drugs may result in competition for protein binding [11].

Recently, molecular biology has affirmed that the cytochrome P450 family of isoenzymes, found in the liver, is responsible for the metabolism of several drugs. Furthermore, the broad range of drugs that undergo cytochrome P450 mediated oxidative biotransformation are involved in a large number of clinically significant drug interactions during concomitant administration $[12,13]$. Research undertaken in this area has uncovered that inhibition based drug interactions constitute the major portion of clinically important drug interactions and that a drug may inhibit the cytochrome P450 isoenzyme whether or not it is a substrate for that isoenzyme. Enzyme inhibition occurs when two drugs sharing metabolism via the same isoenzyme compete for the same enzyme receptor site. The more potent inhibitor will predominate, resulting in decreased metabolism of the competing drug, reduced pharmacological effect and drug toxicity. On the other hand, enzyme induction can result in accelerated enzyme synthesis, faster drug metabolism, sub-therapeutic drug concentrations and potential adverse drug reactions. Nevertheless, the unanticipated effect of both enzyme induction and inhibition, deduced from drug-drug interactions, could lead to severe adverse drug reactions.

The clinical effect of drug-drug interactions varies in terms of severity. While some may range from mild to severe adverse drug reactions, others could be fatal. A case is presented in [14] of a fatal drug interaction caused by ingestion of Clozapine (Clozaril) and Fluoxetine (Prozac). Clozapine is a tricyclic dibenzodiazepine derivative used as an "atypical antipsychotic" in the treatment of severe paranoid schizophrenia. Fluoxetine on the other hand is a selective serotonin reuptake inhibitor (SSRI) used for the treatment of depression. Clinical studies have proven that concomitant administration of Fluoxetine and Clozpine produces increased plasma concentrations of Clozapine and enhances Clozapines pharmacological effects due to suspected inhibition of Clozapine metabolism by Fluoxetine $[14,15]$. In sum, the studies indicated that the combination of these drugs produced lethal concentrations of Clozapine and high therapeutic to toxic concentrations of Fluoxe- 
tine. The deceased suffered from pulmonary edema, visceral vascular congestion, paralytic ileus, gastroenteritis and eosinophillia, all of which are conditions generally associated with Clozapine toxicity [14].

Subsequently, a single report of a drug reaction in a 39-year old woman ultimately contributed to the removal of the allergy drug Seldane (terfenadine) from the market in 1998. Doctors at the National Naval Center in Bethesda, Maryland, admitted the woman to the hospital because of fainting episodes. She had been prescribed Seldane (Terfenadine) 10 days prior. She also started using the prescription drug Nizoral (Ketoconazole) for a vaginal yeast infection. That combination caused potentially fatal changes in her heart rhythm. The Food and Drug Administration (FDA) issued warnings indicating that Ketoconazole interfered with Terfenadine's metabolism, which resulted in increased levels of terfenadine in the blood and slowed its elimination from the body. The FDA also warned that a similar effect could occur if Seldane was taken with antibiotic erythromycin. The drug was taken off the market when Allegra (Fexofenadine), a safer alternative, was approved [16].

Autonomous discovery of drug-drug interaction (DDI) in silico is an area of on-going research that has been explored for decades. Although feasible, the task is far from trivial. When two or more drugs are administered concurrently, in vivo analysis indicates that they tend to have a pharmacologic response beyond that expected from drugs administered individually. The outcome of a drug interaction can be synergistic, antagonistic, or idiosyncratic [17]. When a drug substance interferes with or accentuates the absorption, distribution, or excretion of a second drug, thereby changing the amount and duration of a drug's availability at receptor sites, it produces a pharmacokinetic interaction. A pharmacodynamic interaction, on the other hand, occurs when drugs act on the same receptor, site of action, or physiologic system and thereby have the same physiological effect [17]. 


\subsection{Related Work}

Yildrim et al. [18] proposed the Drug-Target Network and used this to study the relationship between drugs and their protein targets. In their work, a bipartite graph was constructed composed of FDA-approved drugs and proteins connected by drug-target binary associations and clustered based on each drug's Anatomical Therapeutic Chemical classification. Although their study involved discovery of new protein targets for clinical drugs, it was not aimed directly at solving the problem of drug-drug interactions.

Along similar lines, Ma'ayan et al. [19] proposed a drug discovery technique using bipartite graphs, and gene ontology analysis. They developed a bipartite network of FDA approved drugs and their targets and conducted gene ontology analysis on the network in order to draw a connection between pharmacology and computational graph-theoretical Systems Biology. Albeit a promising approach, there is a major limitation preventing any meaningful conclusions to be drawn from the network. That is, the fact that two or more drugs share a common therapeutic target doesn't necessarily imply the existence of an interaction between the two drugs; hence although a link could exist between two or more drugs in the network, a definite or specific interaction between the drugs cannot be determined.

Scheiber et al [20] pursued a global analysis linking chemical features to Adverse Drug Reactions (ADRs). They used datasets of marketed drugs obtained from the PharmaPendium database [21], extracted those that shared common ADRs and toxicities (using only ADR terms stored in the Medical Dictionary for Regulatory Activities (MedDRA)) and identified overlaps of ADR types in chemical space to establish some biological relatedness between the ADRs. According to their work, computing a map of ADRs in chemical space can supposedly help better understand possible ADRs for novel compounds sharing similar substructures.

Campillos et al [22] studied identification of drug targets using side effect similarity. Based on a network of side effect-driven drug-drug reciprocities, they were able to validate drug-target relations experimentally via binding assays. In general, they used phenotypic side-effect similarities to infer whether two drugs share a target, thereby indicating that the use of phenotypic information may be important in infering molecular interactions. 
Zhao et al [23] studied a network-based method for relating pharmacological and genomic spaces for drug target identification. By building a protein-protein interaction (PPI) network, they were able to identify drug-target interactions and also predict biological fingerprints in silico for a number of FDA approved drugs. Their methodology extends the candidate target proteins to a genome-wide scale, which, as a result, enlarges the number of known targets in DrugBank. Similar to the work by Yildrim et al. [18], the study focuses on drug targets and lacks direct inference to drug-drug interactions.

Undoubtedly, the network approach provides a semantic way to organize and analyze enormous databases and also warrants the visualization of nodes in the network whereby a node could serve as an abstract representation of either a drug, a drug target, an adverse reaction, or even a disease [24]. However, in a general sense, these works usually tackle the problem of drug-drug interaction from a single point of view such as via drug ADRs for instance. The problem with this approach is analogous to tackling an obesity problem solely by physical exercises, while ignoring genetics and eating habits amongst other factors. In other words, the mere fact that DDI studies can be conducted from different aspects (ADR, PPI, etc) hints that several factors account for DDIs hence attempting to solve the problem from a single coordinate is not sufficient.

Based on this notion, we devise a way to combine a number of DDI prediction parameters in a multidimensional pharmacological space in order to exercise simultaneous analysis. Our flexible framework permits the expansion of this space to accommodate a plethora of factors easily. Furthermore, rather than simply performing sophisticated statistical analysis on drug data sets such as FDA's AERS database [25] or WHO's Vigibase [26], we present a working model that can support the prediction of potential DDIs amongst newly approved pharmaceutical drugs and even those yet to be approved by the FDA. 


\section{Chapter 3}

\section{Methods}

For a given pharmaceutical drug-pair, we identified biomedical and biochemical characteristics common to both drugs and constructed a dataset using specific attributes such as their chemical compound structure, active ingredients, Human Protein Targets (HPT) and Anatomical Therapeutic Classifications (ATC). Furthermore, we employed a number of similarity measures to establish different levels of relatedness between the pair.

The process of creating the dataset involved collecting drug brand names from the FDA's Structured Drug Label (SPL) files [3]. The SPL is a document markup standard in XML format approved by Health Level Seven (HL7) and adopted by the FDA as a medium for storing and exchanging information. The files contain drug data including but not limited to product and generic names, active and inactive ingredients, ADRs, dosage forms, routes of administration, black box warnings, Drug Enforcement Administration (DEA) schedule and packaging information amongst others. Although all the information provided could be used to uniquely identify a drug, for the purpose of this research, we limited our interest exclusively to brand names, active ingredients and ADRs for each drug.

\subsection{Drug Attributes}

In the process of constructing a dataset for training, we extracted a number of drug attributes from different sources including FDA's SPL files [3] and the DrugBank database [5]. 
The attributes extracted include active ingredients, Anatomical Therapeutic Classification codes, Human Protein Targets and chemical structures. For each drug-pair's active ingredients, we computed a similarity measure using the Jaccard Index and Tanimoto coefficient. We did the same with each drug's set of ATC codes but in addition, we computed a prefix similarity match score that helps identify drugs known to act on the same organs or possess similar therapeutic and chemical classifications. Furthermore, for each drug's set of protein targets, we established scores for direct and indirect interactions between the set elements and protein targets of another drug. Finally, we appended to each drug-pair's feature vector a measure for the similarity between their respective chemical structures. Table 3.2 lists all attributes that comprise any feature vector used in this work. Sections 3.1.1 through 3.1.5 describe each of the attributes and their similarity measures in detail.

\subsubsection{Active Ingredient Similarity}

With active ingredient information successfully extracted, we applied the Jaccard Index as a similarity measure to enable us identify the relationship between two drugs in terms of their chemical make-up. The Jaccard Index measures similarity between two sets and is defined as the cardinality of the intersection divided by the cardinality of the union of the sample sets. We employ this measure to identify the similarity between two drugs given their active ingredients. Suppose the sets A and B represent the active ingredients for drugs A and B respectively, then the Jaccard Index similarity between the two drugs can be computed as

$$
J(A, B)=\frac{|A \cap B|}{|A \cup B|}
$$

Furthermore, since the Tanimoto Coefficient, an extended version of Jaccard Index for binary attributes, takes into account dissimilarity between two sets we include it in the feature space as well. It is defined as:

$$
T(A, B)=\frac{A \cdot B}{\|A\|^{2}+\|B\|^{2}-A \cdot B}
$$




\subsubsection{Anatomical Therapeutic Similarity}

The Anatomical Therapeutic Chemical (ATC) classification system classifies therapeutic drugs. In this classification system, the drugs are divided into different groups according to the organ or system on which they act and their chemical, pharmacological and therapeutic properties. This classification system is maintained by the World Health Organization Collaborating Center for Drug Statistics and Methodology (WHOCC) [27]. According to the classification scheme, drugs are grouped at five different levels. At the first level, the

drugs are divided into fourteen main groups. At the second level they are categorized into pharmacological/therapeutic subgroups. The third and fourth levels are chemical/pharmacological/therapeutic subgroups and the fifth level is the chemical substance. Table 3.1 reveals the complete classification of Metformin as an antidiabetic drug.

\begin{tabular}{l|l} 
ATC Code & Clasification \\
\hline A & Alimentary tract and metabolism (1st lvl: anatomical) \\
\hline A10 & Drugs used in diabetes (2nd lvl: therapeutic) \\
\hline A10B & Blood glucose lowering drugs (3rd lvl: pharmacological) \\
\hline A10BA & Biguanids (4th lvl: chemical subgroup) \\
\hline A10BA02 & Metformin (5th lvl: chemical substance) \\
\hline
\end{tabular}

Table 3.1: Anatomical Therapeutic Chemical classification for the anti-diabetic drug Metformin

\section{Prefix Similarity Match}

By comparing the characters of two ATC codes, we are able to derive a measure of similarity between the two codes. Moreover, repeating the process for all ATC codes for two drugs, we are able to obtain a measure of similarity between them. ATC codes constitute 6 out of the 21 attributes in the dataset. These attributes include:

a) Cardinality of the set of ATC codes for $\operatorname{drug} A$

b) Cardinality of the set of ATC codes for drug $B$, and

c) Therapeutic similarity score for pair-wise comparison of drug $A$ and B's ATC codes.

The similarity between two ATC codes is derived according to their prior probabilities and the probability of their commonality, which is defined as their longest prefix match [23] 


$$
S(i, j)=\frac{2 \times \log (\operatorname{Pr}(\text { prefix }(i, j)))}{\log (\operatorname{Pr}(i))+\log (\operatorname{Pr}(j))}
$$

$\operatorname{prefix}(i, j)$ is the longest matched prefix between ATC code $i$ and $j$. Since a single drug could have several therapeutic functions and hence multiple ATC codes, we define the maximum ATC code similarity as TS:

$$
T S\left(d_{1}, d_{2}\right)=\max _{i \in A T C\left(d_{1}\right), j \in A T C\left(d_{2}\right)}(S(i, j))
$$

$\operatorname{ATC}(d)$ represents all the ATC codes belonging to drug $d$.

In addition, we include a normalized longest prefix match score (atc_lp) to each feature vector by counting the number of prefix matches between two ATC codes for a drug-pair and normalize by the total number of ATC codes between the two drugs. Formally defined as:

$$
L=\frac{\text { prefix }(i, j)}{\left\|A T C\left(d_{1}\right) \cup A T C\left(d_{2}\right)\right\|}
$$

\subsubsection{Protein-Protein Interaction}

Target proteins are biomolecules manipulated by active compounds [28]. Protein binding generally refers to the binding of a drug to proteins in the blood plasma. The amount of drug bound to a protein determines how effective the drug is in the body since the bound drug is kept in the blood stream while the unbound components of the drug may be metabolized. Hence, if a drug is $90 \%$ bound to a binding protein and the remaining $10 \%$ is free, then only $10 \%$ of the drug is active in the system and causing pharmacological effects. However the pharmacological effects of one drug may be altered by another if a protein that can be bound to by both drugs is already saturated by one before the other becomes present. For example, if drug $\mathrm{A}$ is bound to a binding protein and drug $\mathrm{B}$ is unable to bind to that protein, then there would be a higher concentration of unbound drug B. Drug B could also competitively displace drug A from the binding protein, thus raising the unbound fraction 
of drug A and effectively influencing drug A's pharmacological effect. The competition between two drugs to bind a protein is technically referred to as protein-protein interaction (PPI) [29]. If two drugs target similar proteins, then the probability that they will interact is high. Accordingly, the more protein-targets two drugs have in common, the greater the probability of interaction. Based on this, we identified known protein targets for each drug and for each drug-pair, we assign a measure for the number of protein-targets common to both drugs and include it in the feature vector.

\section{PPI Expansion}

Human protein targets constitute 8 out of 21 attributes in the feature space, two of which are cardinalities for the set of proteins each drug targets. Also included is a count for the number of protein targets both drugs have in common and that are known to interact [30]. We represent an interaction between two protein-targets $\alpha$ and $\beta$ as:

$$
L(\alpha, \beta, \lambda)= \begin{cases}\lambda & \text { if a } \lambda \text {-hop PPI exists between } \alpha \text { and } \beta, \lambda \geq 1 \\ 0 & \text { Otherwise }\end{cases}
$$

$\lambda$ is the maximum depth of indirect interaction on the PPI network. When $\lambda=1$, we refer to the interaction as direct. On the other hand, a $\lambda$-hop PPI where $\lambda \geq 2$ is referred to as an indirect PPI. In this work, we restrict the value of $\lambda$ to 3 to ensure that a high level of proximity in terms of closeness between entities is maintained.

For $\lambda \geq 2$, we employ an implication logic model to infer the existence of an indirect interaction between two protein-targets. In the simplest sense, suppose the set of proteintargets for drug $A=\left\{a_{1}, a_{2}, a_{3}, \ldots, a_{n}\right\}, B=\left\{b_{1}, b_{2}, b_{3}, \ldots, b_{m}\right\}$ and $C=\left\{c_{1}, c_{2}, c_{3}, \ldots, c_{q}\right\}$, and $L\left(a_{i}, b_{j}, 1\right)=0$, we can infer a 2 -hop indirect PPI via

$$
\left(\left(a_{i} \Rightarrow c_{r}\right) \wedge\left(c_{r} \Rightarrow b_{j}\right)\right) \Rightarrow\left(a_{i} \Rightarrow b_{j}\right)
$$

where $1 \geq i \leq n, 1 \geq j \leq m$ and $1 \geq r \leq q$. That is, in the absence of a direct interaction between protein targets $a_{i}$ and $b_{j}$, if protein-target $a_{i}$ interacts with $c_{r}$, and $c_{r}$ interacts with 
$b_{j}$, then $a_{i}$ interacts with $b_{j}$, so that $L\left(a_{i}, b_{j}, 2\right)=1$. By including the set of protein-targets for a fourth drug $D=\left(d_{1} d_{2} d_{3} \ldots d_{t}\right)$, with $t \geq 1$, we are able to extend the inference to formulate a 3-hop indirect PPI expansion:

$$
\left(\left(a_{i} \Rightarrow c_{r}\right) \wedge\left(c_{r} \Rightarrow d_{l}\right) \wedge\left(d_{l} \Rightarrow b_{j}\right)\right) \Rightarrow\left(a_{i} \Rightarrow b_{j}\right)
$$

where $1 \geq l \leq t$ Finally, we adopt a closeness measure [23] to define the relative distance between a protein $p$ and a drug $d$ based on the PPI network.

$$
\vartheta_{p d}=\sum_{P_{k} \in T(d)} e^{-L_{p p_{k}}^{2}}
$$

$P_{k}$ is the known target of the given drug $d, T(d)$ denotes the known protein targets of $d$. $\quad L_{p p_{k}} \in\{0,1,2,3\}$ is the shortest distance between $p$ and $p_{k}$ in the PPI network obtained from $L(\alpha, \beta, \lambda)$. $e^{-L_{p p_{k}}^{2}}$ is used to convert protein-protein distance to proteinprotein closeness. This equation denotes that the closeness between drug $d$ and protein $p$ equals the summation of closeness between $p$ and all targets of $d$.

Finally, we define a binary PPI expansion as:

$$
L_{B}(\alpha, \beta)= \begin{cases}1 & \text { if } L(\alpha, \beta, \lambda)>0 \\ 0 & \text { Otherwise }\end{cases}
$$

for which the number of hops leading to a PPI is not accounted for, just the mere fact that a PPI was found is taken into consideration.

\subsubsection{Protein Sequence Alignment}

To further investigate the relationship between two drugs, we provide a third similarity measure between the pair based on their protein target sequences. To do so, we employ the Basic Local Alignment Sequence Tool (BLAST), a rapid sequence comparison tool that uses a heuristic approach to construct alignments by optimizing a measure of local similarity [31, 32]. Since BLAST compares protein and nucleotide sequences much faster than dynamic programming methods such as Smith-Waterman and Needleman-Wunsch [33, 
34], it is widely used for database searches. In this study, we limit our interest to the comparison of two protein sequences at a time, rather than with every protein sequence in the database, hence a complete and time-consuming query as required by the standard BLAST tool is less desirable. As a result, we adopt the BLAST 2 Sequences program, an optimized version of the standard BLAST program which finds multiple local alignments between any two given sequences [35].

Usually, a single drug may have multiple protein targets, hence we sum the scores obtained from the comparison of any two sequences and normalize by the total number of human protein targets for both drugs. For instance, suppose $A=\left(a_{1}, a_{2}, a_{3} \ldots a_{n}\right)$ and $B=\left(b_{1}, b_{2}, b_{3} \ldots b_{m}\right)$ represent the protein targets for drugs $\mathrm{A}$ and $\mathrm{B}$ respectively, $m, n \geq 1$,

and suppose $T\left(a_{i}, b_{j}\right)$ represents the similarity score obtained from BLAST for a pair-wise comparison of protein targets $a_{i}, b_{j}$, taken from drugs $A$ and $B$ respectively, then $S_{T}(A, B)$, the score assigned to the pair is computed as

$$
S_{T}(A, B)=\sum_{i=1}^{n} \sum_{j=1}^{m} T\left(a_{i}, b_{j}\right)
$$

and normalized as

$$
S_{n}(A, B)=\frac{S_{T}(A, B) / \sqrt{S_{T}(A, A)} \sqrt{S_{T}(B, B)}}{|A| \times|B|}
$$

Processing and runtime information is provided in Chapter 4 under Section 4.1.2.

\subsubsection{Chemical Compound Similarity}

By definition, the structure of a chemical compound includes its molecular geometry, electronic structure and crystal structure of molecules. Molecular geometry refers to the spatial arrangement of atoms in a molecule and the chemical bonds that hold the atoms together. Generally, two drugs similar in structure tend to have similar molecular weights and biological activity. The introduction of one in the presence of another could result in unexpected reactions which could either improve or deter their clinical effects. 


\section{Longest Common Substring}

Chemical compound similarity between the chemical structures of both drugs based on the Longest Common Substring (LCS) of each drug's isomorphic chemical structure. LCS is used to compute a global similarity score by computing the ratio between the size of largest common substructure. Given two strings $S$ and $T$, the longest common substring may be found in $O(|S|+|T|)$ time using suffix trees as illustrated in figure 3.2. Structural information for the drugs used in this study was obtained from the KEGG/LIGAND database [36, 37, 38] by mapping each drug's PubChem ID in our database to the respective Entry ID in the KEGG/LIGAND database. Obviously, the more similar two drugs are in terms of their chemical makeup, the closer the cardinality of the LCS would be to the length of either drug, and presumably the more probable it is that the two drugs manifest the same clinical effect on the human body.

For instance, for the drug-pair [Abilify, Vistaril] shown in Figure 3.1., the associated molecular formula for both drugs is given as [C23H27CL2N3O2, C21H27CLN2O22HCL]. From this pair, and ordering from right to left, we can extract the following set of substrings $\{$ "C2", "H27CL", "O2" $\}$ without permutating the set elements, so that the LCS from the set is "H27CL". In the case where the LCS begins with a numerical character, it is simply discarded and the next LCS is accepted if it qualifies. If there are two or more LCSs, any one of them will suffice since only the length of the LCS is relevant and not necessarily the characters in the substring. In the case of Abilify and Vistaril, the normalized LCS score of 0.208 that is finally appended to the feature vector is then computed as:

$$
N_{L C S}(A, B)=\frac{|L C S(A, B)|}{(|A|+|B|)-|L C S(A, B)|}
$$




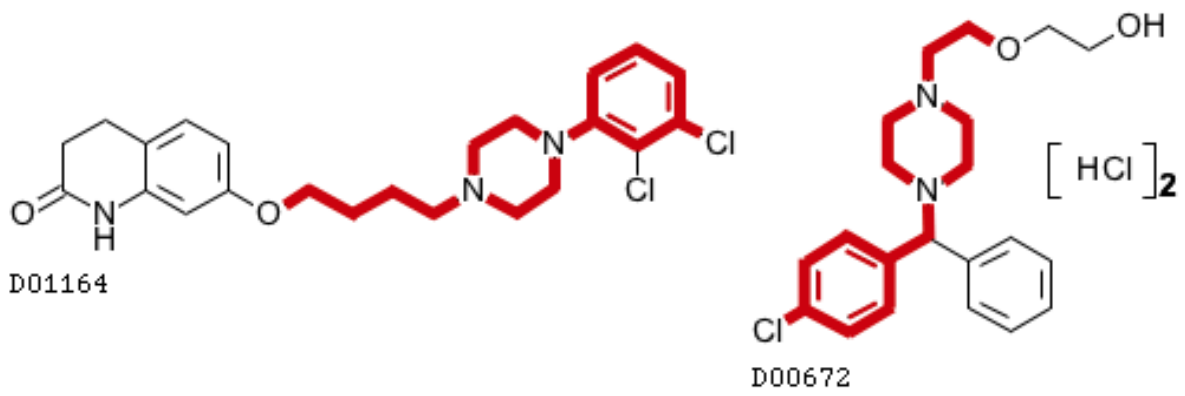

Figure 3.1: Chemical structure similarity for FDA-approved drugs Abilify (KEGG ID: D01164) and Vistaril (KEGG ID: D00672). Longest Common Substring components have been marked with bold lines.

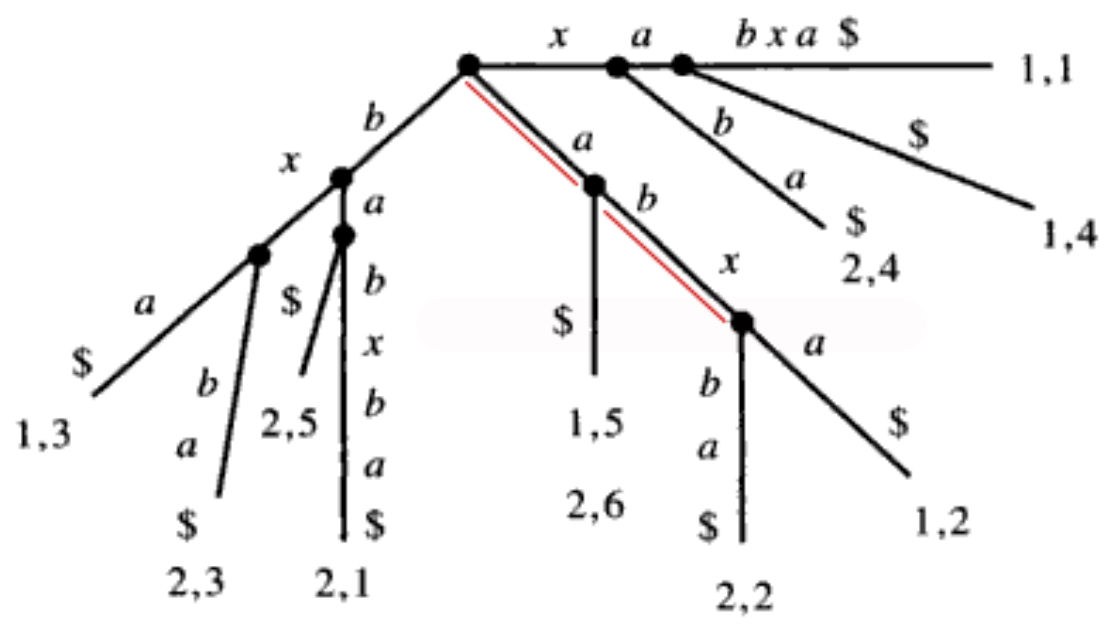

Figure 3.2: Generalized suffix tree for strings $S_{1}=x a b x a$ and $S_{2}=b a b x b a$. The first number at a leaf indicates the string; the second number indicates the starting position of the suffix in that string [1]. Edges marked with double lines highlight the path to the LCS, which is $a b x$ in this case. 


\begin{tabular}{|l|l|l|}
\hline Index & Attribute & Description \\
\hline 1 & pid & Drug-pair Identification \\
\hline 2 & aiA & Cardinality of the set of Active Ingredients for drug A \\
\hline 3 & aiB & Cardinality of the set of Active Ingredients for drug B \\
\hline 4 & ai_c & Number of active ingredients drugs A and B have in common \\
\hline 5 & ai_tc & Active Ingredient Tanimoto Coefficient similarity between drugs A and B \\
\hline 6 & ai_ji & Active Ingredient Jaccard Index similarity between drugs A and B \\
\hline 7 & atcA & Cardinality of the set of ATC codes for drug A \\
\hline 8 & atcB & Cardinality of the set of ATC codes for drug B \\
\hline 9 & atc_c & Number of ATC codes drugs A and B have in common \\
\hline 10 & atc_lp & Normalized Longest Prefix Match between ATC codes (Equation 3.5) \\
\hline 11 & atc_ji & Jaccard Index similarity between a pair of ATC codes \\
\hline 12 & atc_zpm & Prefix Matching (Equation 3.4) \\
\hline 13 & ptA & Cardinality of the set of Human Protein Targets for drug A \\
\hline 14 & ptB & Cardinality of the set of Human Protein Targets for drug B \\
\hline 15 & pt_c & Number of Human Protein Targets drugs A and B have in common \\
\hline 16 & pt_tc & Tanimoto Coefficient for drug A and B's Human Protein Targets \\
\hline 17 & pt_ji & Jaccard Index for drug A and B's Human Protein Targets \\
\hline 18 & ppi & Normalized count of Protein-Protein interactions \\
\hline 19 & ppi_c & Continuous PPI expansion (Equation 3.9) \\
\hline 20 & ppi_b & Binary PPI expansion (Equation 3.10) \\
\hline 21 & blast & BLAST score for drug A and B's protein targets \\
\hline 22 & sfmsim & Chemical Formula Similarity score for the drug pair \\
\hline 23 & class & Class Label \\
\hline
\end{tabular}

Table 3.2: Attributes that constitute a feature vector in the dataset. Owing to the relatively small size of the positive set, drug-pairs comprising known DDIs are assigned IDs between 0 and 1000 while IDs for unlabeled pairs are greater than 1000. 


\subsection{Database Architecture}

Information pertaining to all drugs, their active ingredients, human protein targets and anatomical therapeutic classification was extracted from various datasets and stored in a relational database to allow easy querying of information pertinent to a specific drug. The structure of the database is shown in Figure 3.3. It consists of 8 tables in total of which 4 are independent and the remaining 4 are bridge tables connecting the 2 independent tables.

\subsubsection{Drugs Table}

The drugs table stores brand names for drugs extracted from FDA's SPL files and their PubChem IDs. Also in this table, unique ID numbers are assigned to each brand name.

\subsubsection{Active Ingredients Table}

The active_ingredients table stores information about every active ingredient, for every drug, extracted from the SPL files. In this table, the active ingredients are independent and no association with any drug can be inferred from this table alone. Names are distinct and are assigned unique ID numbers.

\subsubsection{Protein Targets Table}

The protein_targets table stores information about all known human protein targets, for each drug used in this work including their sequence structures. The protein target information was extracted from the DrugBank database [5] for each drug by performing a brand name lookup. Like the Active Ingredients table, the protein targets listed in this table are independent and no association with any drug can be inferred from this table alone. Furthermore, duplicate entries are not permitted and each entry is assigned a unique ID number. 


\subsubsection{ATC Codes Table}

The atc_codes table stores information about Anatomical Therapeutic Chemical classification codes for each drug studied in this work. All ATC codes used in this study were extracted from the DrugBank database [5]. ATC codes in this table are independent, meaning association between drugs and their respective ATC codes cannot be inferred from this table alone.

\subsubsection{Drugs-Active Ingredients Table}

The drugs_ai table serves as a bridge between the drugs table and the active_ingredients table. It permits the querying of which active ingredients can be found in a given drug simply by executing the SQL statement

SELECT name FROM active_ingredients

WHERE_id IN (SELECT ai_id FROM drugs_ai

WHERE drug_id $=($ SELECT_id FROM drugs WHERE name $=$ 'x' $)$ )

where $x$ is the drug whose set of active ingredients is to be determined.

\subsubsection{Drugs-Protein Targets Table}

The drugs_pt table acts as a bridge connecting the drugs table to the protein_targets table. If permits the querying of which proteins are targeted by a specific drug by executing the SQL statement

SELECT name FROM protein_targets WHERE_id IN (SELECT pt_id FROM drugs_pt WHERE drug_id $=($ SELECT_id FROM drugs WHERE name $=$ 'x' $)$ ) where $x$ is the drug whose set of protein targets is to be determined.

\subsubsection{Protein-Protein Interaction Table}

The ppi table contains a list of protein-pairs known to interact. Information about proteinprotein interaction was obtained from the BigGrid database [30]. Protein-target IDs in this 
table reference protein-target IDs in the protein_interactions table.

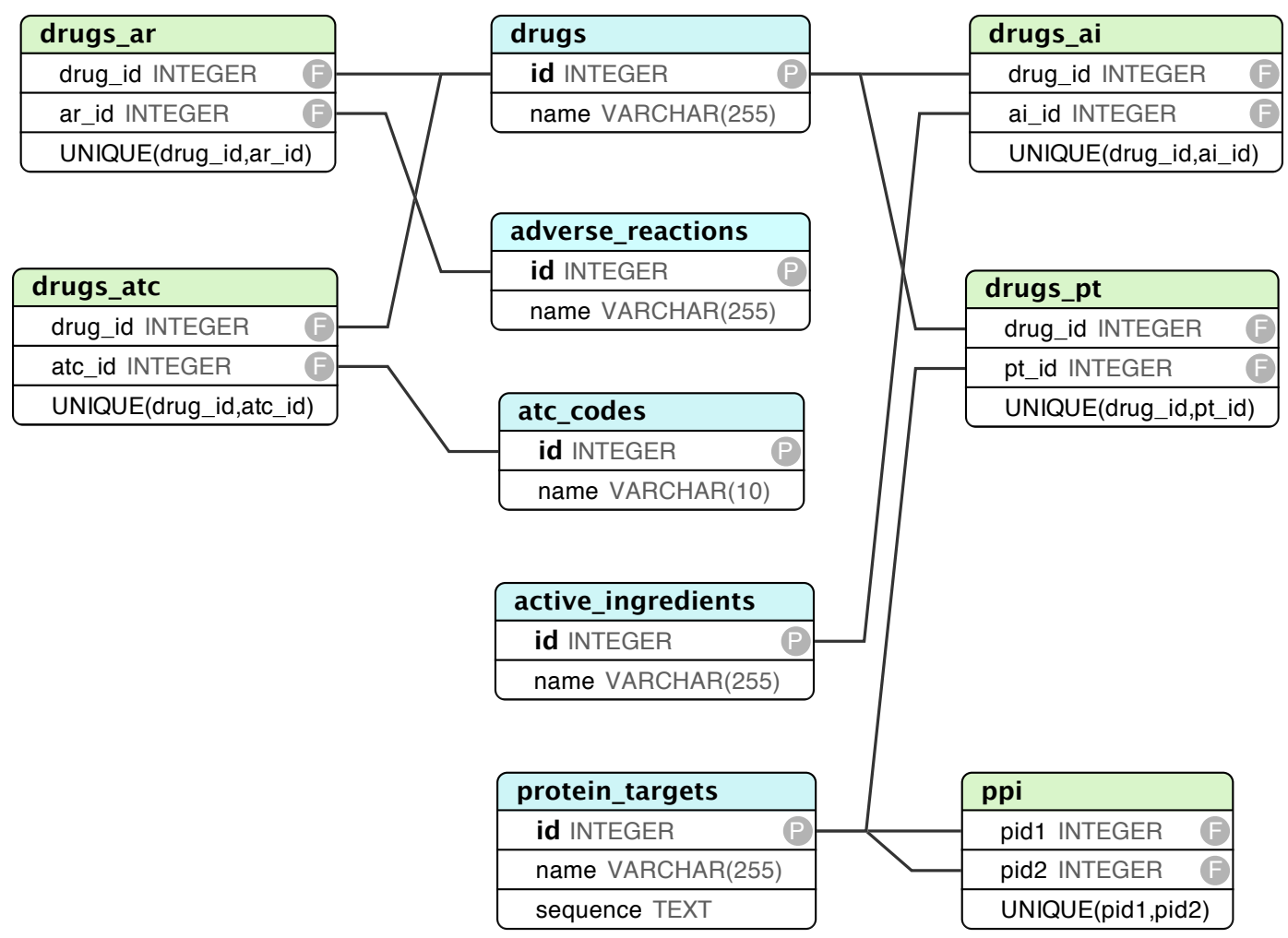

Figure 3.3: Drug Database Architecture. The tables drugs_ar, drugs_atc, drugs_ai, drugs_pt, and ppi serve as bridges to the drugs, adverse_reactions, atc_codes, active ingredients and protein_targets tables. Although we deliberately omitted ADR information from our dataset, we later used it to prove the credibility of our system in Section 4.3. 
Drugs with most ATC classifications
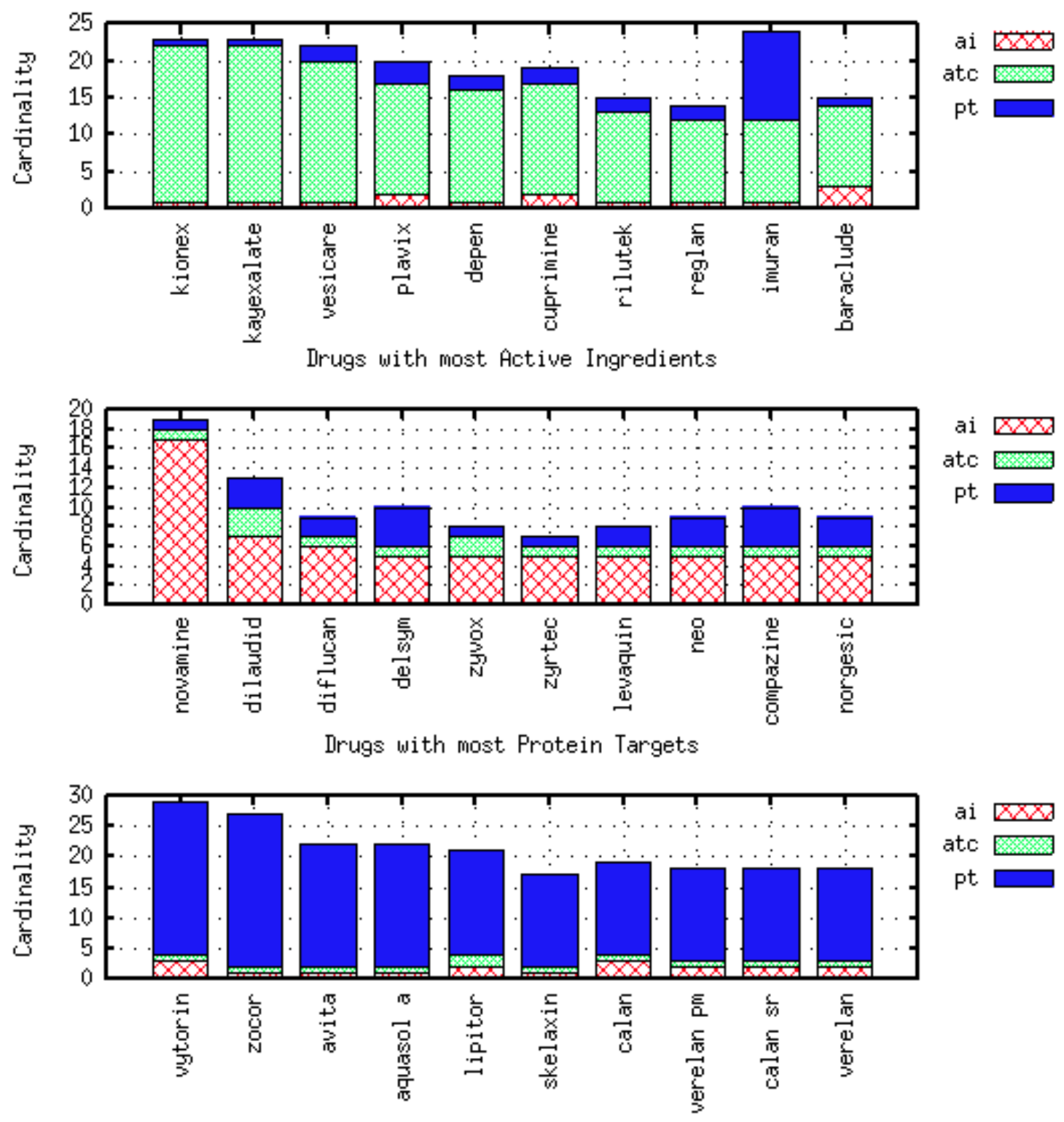

Figure 3.4: Top 10 drugs from the drug database with the most active ingredients, most ATC classifications and most human protein targets. 
Figure 3.4 shows some statistics of sample drugs in our drugs database. Notably, no drug appeared in the top 10 for more than one attribute implying that the presence of one attribute does not necessarily dictate the presence of another. In other words, an increase in the number of active ingredients, for example, does not directly imply an increase in the number of human protein targets for the drug, since if that was the case, then the statistics visualized would have included at least one drug common to all three sets. Nevertheless it is equally likely that the attributes are inversely related. That is, hypothetically, the more active ingredients found in a drug, the fewer human proteins the drug is likely to target and vice versa. Hence, clearly the relationship between these attributes is non-trivial. The complete distribution of drug-attribute cardinalities that can be found in our database has been visualized in Figure 3.5.
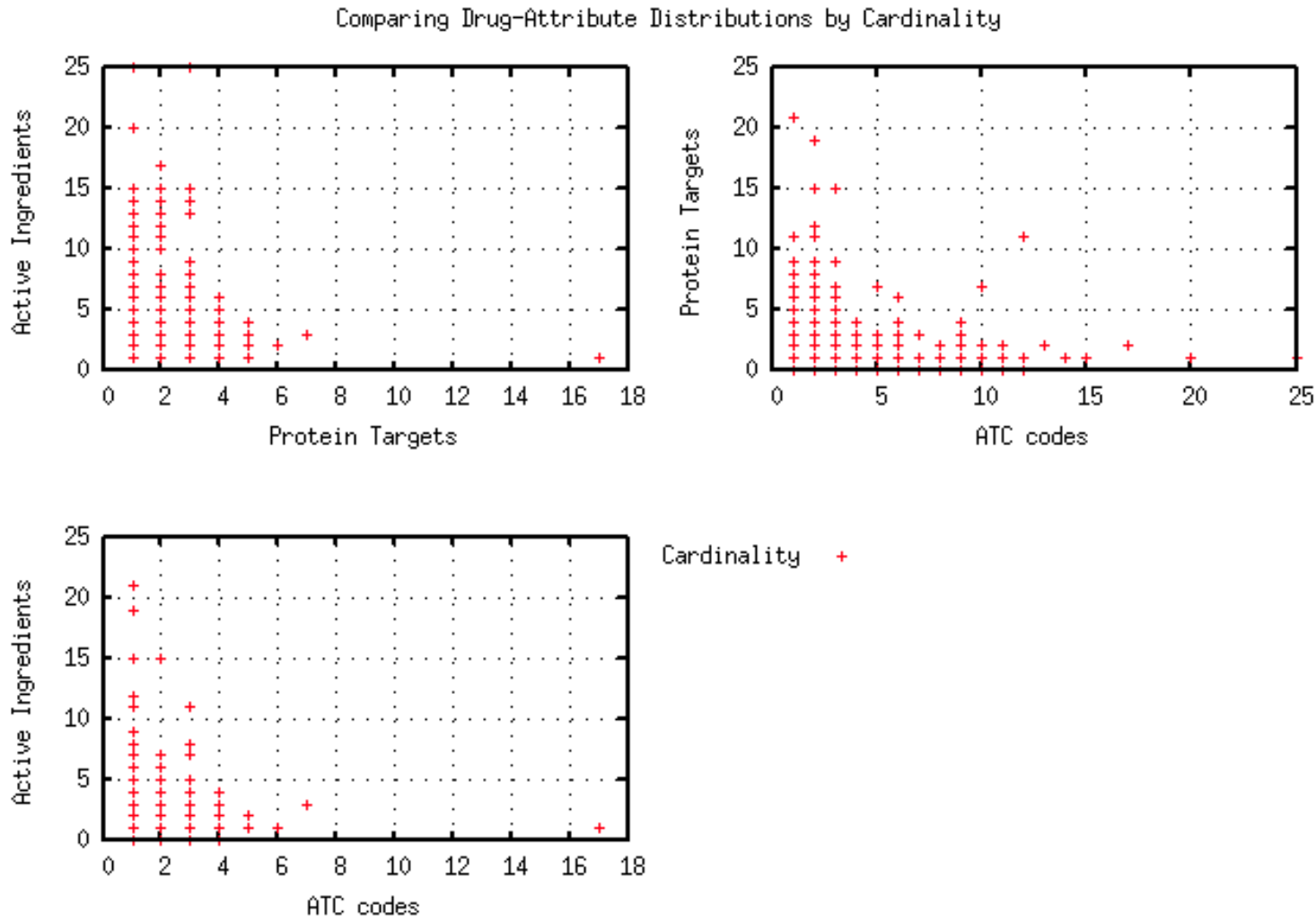

Figure 3.5: Distribution of drug-attributes stored in the database within which all drugs studied in this work are stored. 


\subsection{PU-Learning}

Traditional supervised learning classification algorithms train on datasets whose class labels are explicitly known. In reality however, the availability of such datasets is scarce. For a given dataset, if its class labels are unknown, then unsupervised learning techniques such as data clustering would suffice. Admittedly, unsupervised learning is beneficial if none of the classes in the dataset are known prior to classification, even though the results obtained are typically not at par with supervised learning classifiers. In sum, researchers either know of all classes in a dataset prior to their experiments or none at all. Between the two extremes one employs semi-supervised learning techniques.

Given the nature of the dataset used in this study, specifically with reference to the lack of negative instances due to the difficulty in confirming or establishing a certainty that two drugs do not interact, we employed a partially supervised learning technique called PositiveUnlabeled Learning (or PU-Learning for short) [39, 40, 41, 42, 43]. PU-Learning involves learning from Positive (P) and Unlabeled (U) examples. The main algorithm involves 2 steps:

Step 1: Identify a reliable set of negative examples RN, from the given unlabeled dataset U using techniques such as Naïve Bayesian approach, spy technique, 1-Disjunctive Normal Form (DNF) and Rocchio algorithm [42]. In this work, we employed Naïve Bayes as the primary extractor of Reliable Negative instances from U because of its strong independence assumptions used in classification.

Step 2: Build a set of classifiers by applying a classification algorithm iteratively using the given labeled positive examples $\mathrm{P}$, the extracted negative examples $\mathrm{RN}$ and the remaining unlabeled instances U-RN. Finally, select a good classifier from the set. For this step, we trained three classifiers: Support Vector Machine (SVM), J48 Decision Tree, and K-Nearest Neighbors (KNN) simultaneously using a modified version of the Co-Training algorithm [44] called Tri-Training [45]. In Tri-training, the learning process of each classifier is greatly influenced by the other two classifiers, akin to a majority-vote scheme. Collectively, the two steps summarized above make up the Expectation Maximization (EM) Algorithm. Section 3.3.1 elaborates on this algorithm. 
Unfortunately, PU-Learning performs rather poorly when $\mathrm{P}$ is relatively small compared to U. This drawback is circumvented by the Positive Document Enlarging PU Classifier (PE-PUC) [43], a slightly modified PU-Learning protocol that is distinguished by a crucial Entity Set Expansion step. This step involves expanding the size of $\mathrm{P}$ by extracting Reliable Positive (RP) instances from U-RN. Customized to suit the nature of our work, we combined the Naïve Bayes approach and the spy technique $[39,46]$ in order to obtain a set of Reliable Negative drug-pairs. Generally, this would be interpreted as drug-pairs that do not interact. Acknowledgement of this interpretation is erratic, especially since a $100 \%$ certainty of the fact is nearly impossible. Therefore, within the scope of this work, RN encapsulates drugs whose attributes when combined to determine similarities, exhibit patterns that are emphatically distinguishable from proven drug-drug interactions. 


\subsubsection{EM/S-EM Algorithm}

The Expectation Maximization algorithm is a popular class of iterative algorithms for maximum likelihood estimation in the presence of unlabeled data. Generally, it is used to probabilistically assign values to unlabeled instances in a corpus by computing the expected value for each missing value [39]. The EM algorithm consists of two steps, the Expectation step and the Maximization step. Generally, the Expectation step simply fills in the missing data, after which parameters are estimated by the Maximization step.

In order to obtain reliable information for identification, the Expectation step is altered to include the act of spying, hence the name S-EM. Spying typically involves migrating $s \%$ randomly selected "spy" instances, $S$, from the positive set $P$ to the unlabeled set $U$. The instances in $S$ are collectively referred to as spies. By adding $S$ to $U$, positive instances in $U$ can easily be inferred and extracted since they would exhibit characteristics and behavior

of instances in $S$. For our experiment, we used a sampling ratio $s=15 \%$ to generate the spy set $S$.

The spying technique enables us to extract both Positive and Negative examples from $U$, respectively referred to as Reliable Positive $(R P)$ and Reliable Negative $(R N)$ instances. The subsample $P \cup R P \cup R N$ can then be used to efficiently construct a classification model in the Maximization step. Figure 3.6 shows the general procedure. 

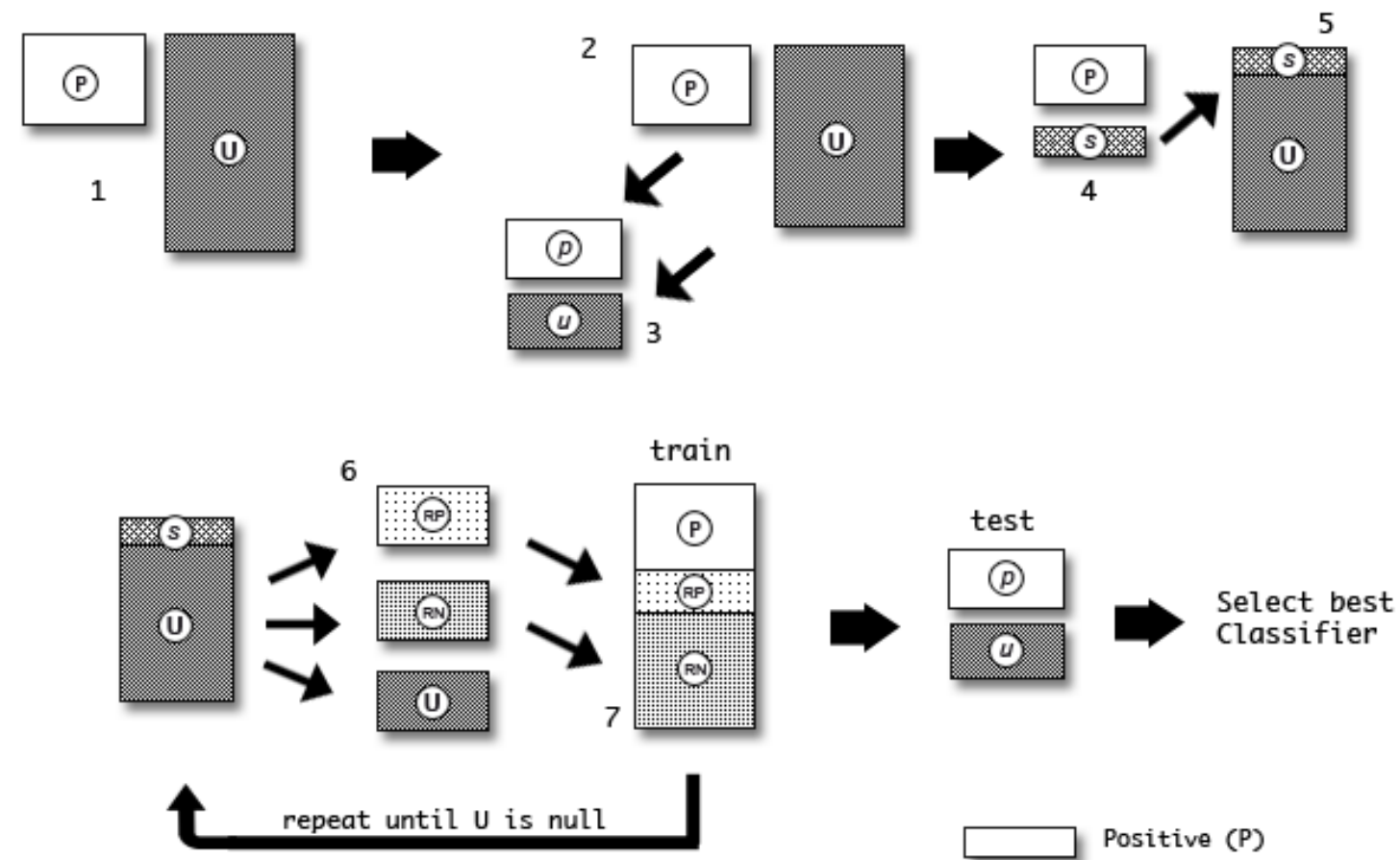

\begin{tabular}{|c|c|}
\hline & Positive (P) \\
\hline & Unlabeled (U) \\
\hline 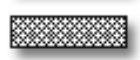 & Spies (s) \\
\hline mon & Reliable Positive (RP) \\
\hline 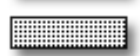 & Reliable Negative (RN) \\
\hline
\end{tabular}

Figure 3.6: PU-Learning procedure, showing incorporation of the spy technique with the EM Algorithm.. 


\subsubsection{Co-Training}

Co-training involves the training of two distinct classifiers on different attributes of the same instance [44]. That is, for a given instance $x=\left(x_{1}, x_{2}\right)$, two classifiers $h_{1}$ and $h_{2}$ are trained on $x_{1}$ and $x_{2}$ respectively. From the unlabeled set $U$, the instances most confidently classified by both classifiers as positive and negative are added to the labeled set $L$.

\subsubsection{Tri-Training}

The Tri-training algorithm $[47,45]$ incorporates the $E$ step from the EM algorithm and a modified version of the idea of Co-Training in order to accommodate a third classifier. In the original algorithm, three SVM classifiers were used. The reason for choosing SVM as the primary classifier was not explicitly discussed, however, after training, the best of the three SVM classifiers is returned.

In our experiment, we follow the logic behind Tri-training very closely, yet, rather than training three instances of the same classifier, we train three distinct classifiers and return the best of the three, since the performance of either classifier could greatly be influenced by both the training data and the parameters used. The modified Tri-training algorithm is outline in Algorithm 1. 


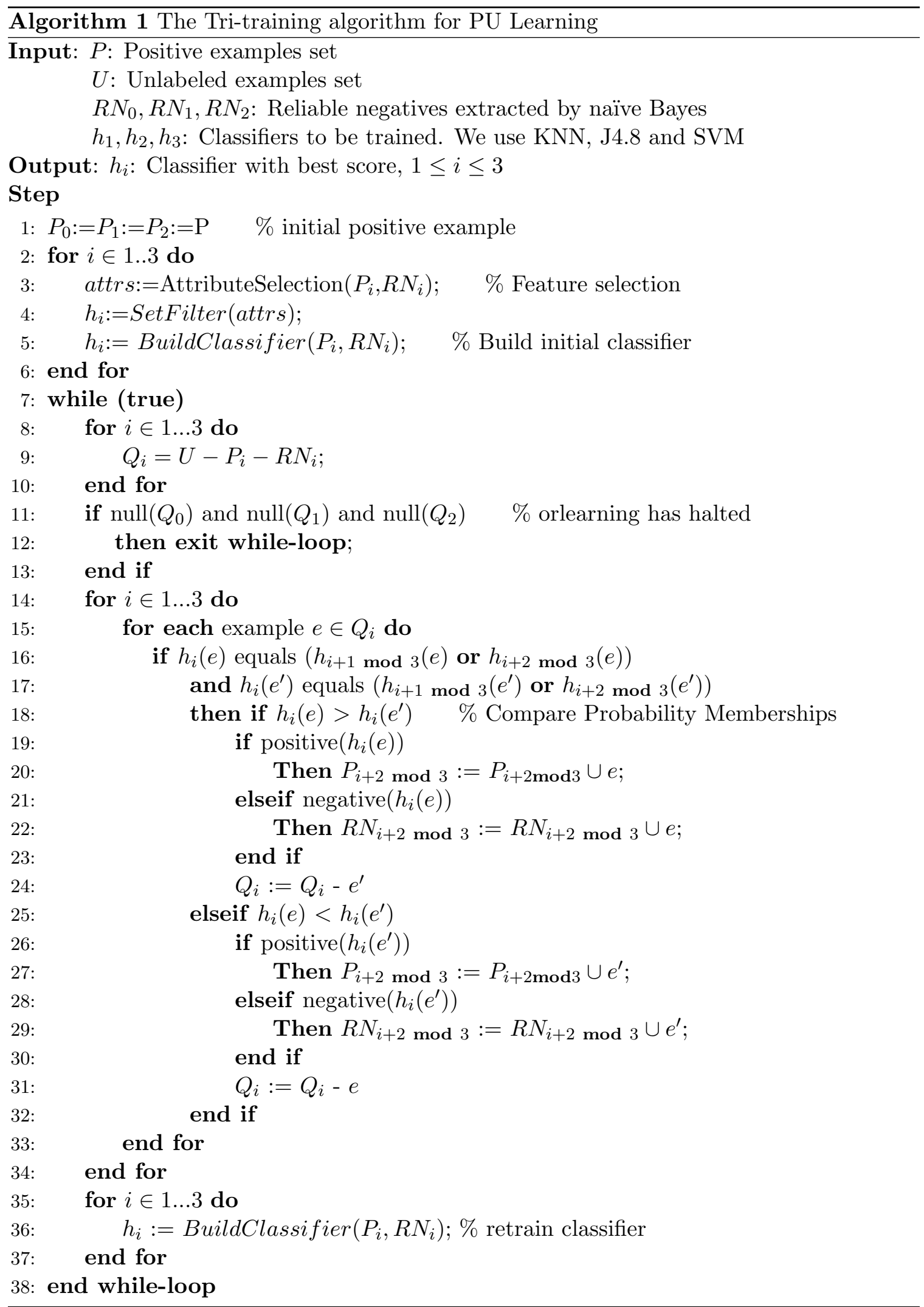


The algorithm accepts four sets of parameters, namely the set of Positive instances, the set of Unlabeled instances, the set of Reliable Negative instances extracted using a Naïve Bayes classifier, and the set of classifiers to be trained, specifically, k-Nearest Neighbors (with $k=7$ ), J48 decision tree, and Support Vector Machines (SVM) [48].

The first step of the Tri-training algorithm involves the initialization of three sets of Positive instances: $P_{0}, P_{1}$ and $P_{2}$ (line 1$)$. The algorithm then builds the initial classifiers $h_{1}, h_{2}$, and $h_{3}$ by first selecting the most influential attributes and filtering the dataset, retaining only the attributes that were selected (line $2-6)$. The next step involves the main loop (lines $7-38$ ) which commences by removing each set of Positive $\left(P_{i}\right)$ and Reliable Negative $\left(R N_{i}\right)$ instances from the set of Unlabeled instances in order to obtain three sets of unlabeled instances $\left(Q_{i}\right)$ to be used in further training of the three classifiers (lines $\left.8-10\right)$, and terminated when $Q_{i}$ is empty (or subsequent removal of $P_{i}$ and $R N_{i}$ from $U$ produces the same $Q_{i}$ as in the previous iteration) (lines $11-13$ ). If $Q_{i}$ is non-empty, the algorithm proceeds to the training step (lines $14-34$ ) where the classification of an example $e$ in $Q_{i}$ by a classifier $h_{i}$ is dependent on a majority vote (i.e. in concurrence with at least one other classifier). If the classification of both $e$ and its inverse $e^{\prime}$ obtain a majority vote, then the example $\left(e\right.$ or $\left.e^{\prime}\right)$ that is classified with the highest probability of membership (or confidence) is appended to either the Positive set $P_{i}$, if it was classified as positive, or to the Reliable Negative set $R N_{i}$ otherwsise (lines $15-33$ ). This is how the algorithm handles the inverse feature vector problem. Although not outlined in the pseudocode, during the training process, we keep records of each classifier's F-measure computed per iteration and after 20 iterations, return the classifier with the highest average F-measure as the best classifier.

Figure 3.6 provides a generalized overview of PU-Learning without the details of the algorithmic steps. Step 1 indicates identification and separation of the Positive $(P)$ and Unlabeled $(U)$ sets of data. In Steps 2 and 3, a portion of both $P$ and $U$ is allocated for testing and the remainder is used to training. Steps 4 and 5 depict removing a fraction of instances from the Positive training set to act as spies (s) and appending this set to $U$. In Step 6, Reliable Positive $(R P)$ and Reliable Negative $(R N)$ instances are extracted from $s \cup U$ and concatenated with the Positive training set $(P)$. This step is repeated until no 
new $R N$ instances can be extracted. The learners are then trained one last time using the dataset $P \cup(R P \cup R N)$ and test on the allocated test set. The best classifier is then selected after evaluating classification performance on the test set.

The Tri-training algorithm has a computational complexity of $O\left(N^{2}\right)$. This is primarily due to the fact that the main loop is only terminated when $U$ is either null or learning has halted, which means that all possible $R N$ instances that can be extracted from $U$ have been extracted. Therefore in the worst case, a single $R N$ instance is extracted for each of the $N$ iterations, where $N$ is the cardinality of $U$. If that is the case, then the main loop (lines $7-38$ ) will be executed $N$ times and be restarted $N$ times after rebuilding the classifiers, yielding a worst case runtime of $O\left(N^{2}\right)$. 


\section{Chapter 4}

\section{Experiment}

\subsection{Experimental Setup}

\subsubsection{Data Sets}

We constructed a dataset:

$$
D=\left(x_{i}, y_{i}\right) \mid x_{i} \in R^{p}, y_{i} \in-1,1_{i=1}^{n}
$$

where $y_{i}$ is either 1 or -1 , indicating the class to which $x_{i}$ belongs. Each $x_{i}$ is a pdimensional vector, where $p=23$, representing the number of drug attributes (including drug-pair ID and class label).

From the 1,300 unique drugs extracted from FDA's SPL files, we paired each drug with all others in the list and produced 1,688,700 unique drug-pairs. The inclusion of each pair's feature vector produces a $1,688,700 \times 21$ matrix $(1,688,700 \times 19$ matrix if the drug-pair ID and class label are excluded), which represents the dataset used in this study. For 412 of these drug-pairs, we were able to confirm drug-drug interactions (DDIs) via Stockley's Drug Interactions [4] and general online sources. These drug-pairs were labeled as positive and served as our 'Gold Standard' dataset. The remaining 1,686,088 drug-pairs were left unlabeled. After running BLAST queries on the drug-pairs, we were able to obtain scores 
for 397 positive instances and 1,587,397 unlabeled instances.

\subsubsection{Computational Environment}

We forked 21 UNIX terminal processes to execute BLAST 2 on 1,688,700 drug-pairs ( $(n-$ 1), where $n=1,300$ ) with the first 20 terminals processing 80,000 pairs and the last terminal processing 90,000 pairs on a 2010 Macbook Pro equipped with a $2.4 \mathrm{GHz}$ Intel Core 2 Duo processor, 4 GB $1067 \mathrm{MHz}$ DDR3 memory chip and $1.07 \mathrm{GHz}$ Bus Speed. Processing all the drug-pairs took approximately 3 weeks to complete at a rate of $0.2 \%$ per hour per terminal (and all-together as a matter of fact due to parallelization), providing us with protein sequence alignment scores for at least 397 positive drug-pairs and 1,587,397 unlabeled drug-pairs. Mathematical analysis indicates that sequential execution of BLAST 2 on all 1,688,700 drug-pairs on the same hardware could have taken up to 63 weeks (15 months) to complete.

All pre- and post-processing scripts were written in Python.

GNUPLOT served as the main graphing utility, and

Cytoscape provided tools for visualization of all DDI networks.

Version numbers and additional information pertaining to Python, GNUPLOT and Cytoscape can be found in Appendices A.1, A.2 and A.3 respectively.

\subsubsection{Data Pre-processing}

Generally, pre-processing of data used in data-mining and machine learning involves cleaning the data to avoid learning a classifier on erroneous or inconsistent information such as $\{$ alive $:$ yes, deceased $:$ yes $\}$ or $\{$ weight : 180, height: -6$\}$. A dataset that contains such values should not be trained on and failure to identify and remove such information from a dataset would result in a poorly trained, inconsistent and unreliable classifier.

Although the dataset constructed and used in this work does not contain such erroneous data, it is comprised of duplicate instances and continuous data which equally affects the performance of certain classifiers when learned on, hence instance selection and discretization turned out to be crucial pre-processing procedures in this work. 


\section{Discretization}

Continuous values within a dataset for a given feature can be converted to discrete values by means of discretization. Discretization is necessary prior to building a classification model because not only is it impossible for certain classifiers to accept continuous values as input but also certain attributes in a dataset may contain so many values that the classification algorithm cannot easily identify interesting patterns from which to create a model. Well-known classifiers such as Support Vector Machines, Random Forests and even Naive Bayes tend to exhibit better performance when trained on discretized variables rather than continuous, attributable to their sensitivity to the dimensionality of the data.

Discretization is the process of putting values of a column into bins so that there are a limited number of possible states. Assuming each bin is assigned a unique ID with respect to the total number of buckets, then a continuous value can simply be replaced by the bin ID. This method of discretization referred to as Binning.

Overall performance of a classification model involving discretization can greatly be influenced by the total number of bins used. Decision on the number of bins to use can be made by defaulting to a specific value, such as 5 or 10 , or by computing $\sqrt{n}$, where $n$ is the number of distinct values in the column.

Out of the 19 attributes contained in the dataset used in this work, 7 consist of continuous values. These are specifically the Tanimoto Coefficient and Jaccard Index related attributes, normalized similarity counts, ATC Prefix Matching scores, BLAST scores, and Protein-Target expansions. For each column to be discretized, we set the number of bins to $\sqrt{n}$ if $(\sqrt{n}<10)$, otherwise we defaulted to 10 bins. 


\subsection{Training}

The training process involved applying the PU Learning algorithm on the dataset constructed with feature vector attributes discussed in section 3.2.

\subsubsection{Performance Measures}

Evaluation measures used in this work and as illustrated in Figures 4.1 and 4.2 are Precision, Recall and F-measure, with each defined as:

$$
\begin{gathered}
\text { Precision }=\frac{\mathrm{TP}}{\mathrm{TP}+\mathrm{FP}} \\
\text { Recall }=\frac{\mathrm{TP}}{\mathrm{TP}+\mathrm{FN}} \\
\text { F-measure }=2 \times \frac{\text { Precision } \times \text { Recall }}{\text { Precision }+ \text { Recall }}
\end{gathered}
$$

Precision represents the number of correct positive predictions per positive predictions while Recall represents the number of correct positive predictions per positive examples. TP is the number of True Positives while FP and FN represent the number of False Positives and False Negatives respectively. These metrics enable us to effectively evaluate the competence of our classifiers with respect to the ground truth. 


\subsection{Results}

\subsubsection{Training}

\section{Instance Selection}

The first step we took to prepare the data for mining was to identify and discard duplicate instances. This was achieved by ignoring the first and last columns that store the instance ID and class labels respectively, leaving only drug-pair attributes to used for comparison. The removal of duplicates left behind 363,277 unique unlabeled instances. We then proceeded to remove instances with attributes similar to those in the positive set so as to avoid class-label inconsistencies among similar feature vectors and in turn avoid confusing the classifier. Not doing so would mean an instance could be positive and unlabeled at the same time. And being unlabeled, as far as a classifier is concerned, could very well mean that the instance could be negative, hence a contradiction. Learning on such data is likely to produce a very poor classifier.

We then identified the most influential number of instances (of reasonable size) that should be used in training. From Figure 4.1, it can be noted that training with 700 instances yields the best results and since 700 is approximately twice the number of positive instances, it suffices. 

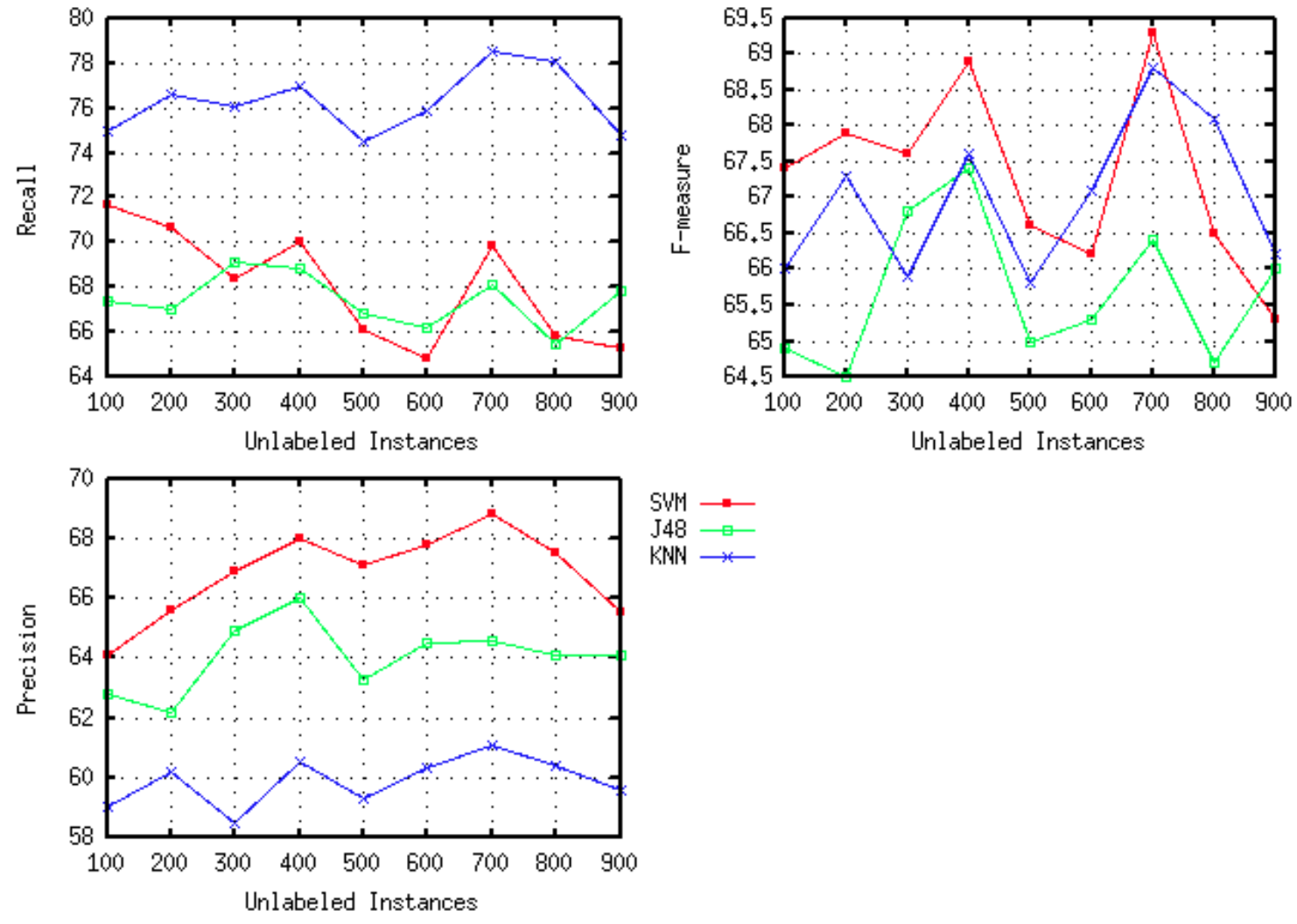

Figure 4.1: Influence of the size of unlabeled data on PU Learning using all 21 attributes. The best output is realized when 700 unlabeled instances are used in training. 


\section{Feature Selection}

Feature selection is a dimensionality reduction technique used to decrease the time taken to train classification models and occasionally improve classification. Also known as subset selection, the primary motive is to select a subset of input variables among an array of others by means of eliminating those with little relevance in terms instance-class prediction. Feature selection can significantly improve the learning of a classifier and increase its potential for correctly classifying unseen data. A number of algorithms have been devised for feature selection such as Forward Selection, Backward Selection, Sequential Forward Selection, Sequential Backward Selection and InfoGain.

In this work, we adopted Sequential Backward Selection as the Feature Selection algorithm. Table 4.1 shows the attribute's index and name as specified in Table 3.2, the feature subset selection (FSS) score, number of time selected out of the 20 runs, and the statistical

analysis metrics F-ratio and p-value. As discussed in Section 4.3.3, if $P \leq 0.05$, the null hypothesis may be rejected. In general categories selected are drug ATC codes, proteinprotein interactions, human protein targets and active ingredients, however with rejection of the null hypothesis, the main attribute category selected is human protein targets 


\begin{tabular}{|l|l|l|l|l|l|}
\hline Index & Attribute & FSS Score & Frequency & F-ratio & p-value \\
\hline 20 & ppi_b & 0.57 & 1.00 & 7.98 & 0.000560 \\
\hline 13 & ptA & 0.56 & 1.00 & 5.50 & 0.005193 \\
\hline 7 & atcA & 0.38 & 1.00 & 4.93 & 0.008748 \\
\hline 14 & ptB & 0.36 & 0.95 & 4.10 & 0.018923 \\
\hline 22 & sfmsim & 0.28 & 0.95 & 2.91 & 0.058532 \\
\hline 8 & atcB & 0.54 & 1.00 & 2.52 & 0.084606 \\
\hline 12 & atc_zpm & 0.96 & 1.00 & 3.33 & 0.039211 \\
\hline 10 & atc_lp & 0.94 & 1.00 & 2.13 & 0.123846 \\
\hline 15 & pt_c & 0.74 & 0.55 & 1.58 & 0.210207 \\
\hline 17 & pt_ji & 0.27 & 0.55 & 1.01 & 0.368922 \\
\hline 4 & ai_c & 0.73 & 0.55 & 1.03 & 0.358883 \\
\hline 9 & atc_c & 0.38 & 0.55 & 0.43 & 0.654283 \\
\hline 11 & atc_ji & 1.12 & 0.35 & 0.48 & 0.619021 \\
\hline 19 & ppi_c & 0.6 & 0.65 & 1.75 & 0.178261 \\
\hline 5 & ai_tc & 0.18 & 1.00 & 0.88 & 0.416748 \\
\hline
\end{tabular}

Table 4.1: Top attributes selected during the feature-selection process. Each value is an average of 20 runs.

Evaluation of SVM, J48 and KNN using varying sets of feature-subset selected attributes is provided in Figure 4.2. For each iteration, the top $n$ attributes returned by the featureselection algorithm are used in training on 700 randomly selected unlabeled instances, where $2 \leq \mathrm{n}<22$. Notably, the best results are obtained when 16 attributes are used to train SVM in terms of Precision and F-measure averaging approximately $71 \%$ 

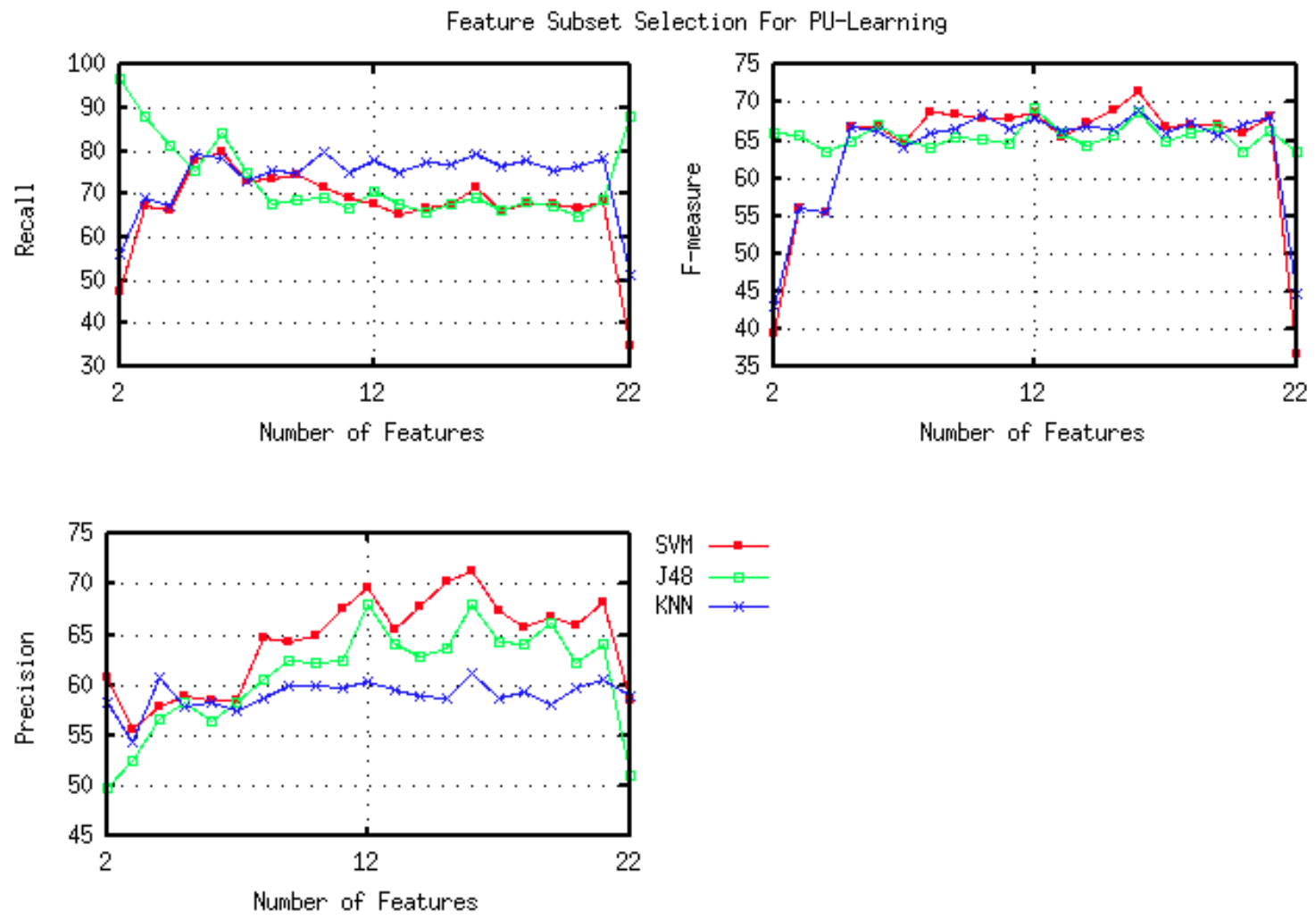

Figure 4.2: Impact of feature subset selection on 700 unlabeled instances. 


\subsubsection{Testing}

Evaluation of the test results produced by the SVM classifier has been provided in Figure 4.3. Each measure is an average of 100 runs on a test set of 200 instances (100 positive, 100 unlabeled) randomly selected during each iteration. As confidence is increased from $0 \%$ to $100 \%$, Precision drops from $75 \%$ to $20 \%$ while Recall increases from $65 \%$ to $71 \%$. An equilibrium of $65 \%$ is realized between the two metrics and their F-measure at a confidence of $50 \%$. No test instances were classified at a confidence of $100 \%$ therefore Recall is $0 \%$ and Precision cannot be computed.

In addition, a DDI network showing known interactions and probabilistic interactions predicted by the SVM classifier has been illustrated in Figure 4.4. Potential DDIs in the network comprise interactions predicted by our system with confidence $\geq 98 \%$.

To further evaluate the applicability of our system, we obtained a list of medications prescribed to 20 patients at the Chestnut Ridge Psychiatric Hospital located in Morgantown, West Virginia. For each patient's medication list, we paired the drugs and passed them to our system, which then flagged potential interactions among a number of pairs found in 9 out of 20 medications lists. We then queried the Adverse Event Reporting System (AERS) database [25] for reported events involving any of the drug-pairs predicted to interact and actually found cases for DDIs predicted in 6 out of 9 medication lists. The results from this experiment have been tabulated in Tables 4.2 and 4.3 .

Finally, we provide a list of potential DDIs predicted by our system involving psychotropic medications retrieved from the dataset employed in this study. The complete list can be found in Table 4.4 along with the level of confidence on which the prediction is based. 


\begin{tabular}{|c|c|c|c|}
\hline Patient & Drug A & Drug B & Confidence \\
\hline \multirow[t]{2}{*}{2} & proair (ALBUTEROL) & flexeril (CYCLOBENZAPRINE) & 98.78 \\
\hline & flexeril (CYCLOBENZAPRINE) & accuneb (ALBUTEROL) & 97.18 \\
\hline \multirow[t]{5}{*}{5} & zocor (SIMVASTATIN) & prozac (FLUOXETINE) & 84.23 \\
\hline & zocor (SIMVASTATIN) & sarafem (FLUOXETINE) & 84.23 \\
\hline & zocor (SIMVASTATIN) & vistaril (HYDROXYZINE) & 81.23 \\
\hline & zocor (SIMVASTATIN) & namenda (MEMANTINE) & 64.62 \\
\hline & sarafem (FLUOXETINE) & atarax (HYDROXYZINE) & 55.82 \\
\hline \multirow[t]{2}{*}{7} & anafranil (CLOMIPRAMINE) & toprolxl (METOPROLOL) & 93.07 \\
\hline & lopressor (METOPROLOL) & anafranil (CLOMIPRAMINE) & 72.91 \\
\hline \multirow[t]{10}{*}{8} & tricor (FENOFIBRATE) & klonopin (CLONAZEPAM) & 85.21 \\
\hline & tricor (FENOFIBRATE) & glucophage (METFORMIN) & 81.69 \\
\hline & tricor (FENOFIBRATE) & riomet (METFORMIN) & 81.69 \\
\hline & triglide (FENOFIBRATE) & klonopin (CLONAZEPAM) & 78.77 \\
\hline & plavix (CLOPIDOGREL) & riomet (METFORMIN) & 61.82 \\
\hline & plavix (CLOPIDOGREL) & fortamet (METFORMIN) & 58.16 \\
\hline & plavix (CLOPIDOGREL) & lofibra (FENOFIBRATE) & 55.63 \\
\hline & plavix (CLOPIDOGREL) & tricor (FENOFIBRATE) & 55.63 \\
\hline & plavix (CLOPIDOGREL) & antara (FENOFIBRATE) & 54.11 \\
\hline & zyprexa (OLANZAPINE) & plavix (CLOPIDOGREL) & 50.00 \\
\hline \multirow[t]{8}{*}{11} & riomet (METFORMIN) & cogentin (BENZTROPINE) & 88.88 \\
\hline & cogentin (BENZTROPINE) & glucophage (METFORMIN) & 83.28 \\
\hline & cogentin (BENZTROPINE) & riomet (METFORMIN) & 83.28 \\
\hline & cogentin (BENZTROPINE) & fortamet (METFORMIN) & 73.56 \\
\hline & depakote (DIVALPROEX) & fortamet (METFORMIN) & 68.44 \\
\hline & klonopin (CLONAZEPAM) & vistaril (HYDROXYZINE) & 64.51 \\
\hline & vistaril (HYDROXYZINE) & glucophage (METFORMIN) & 60.31 \\
\hline & atarax (HYDROXYZINE) & riomet (METFORMIN) & 51.88 \\
\hline \multirow[t]{10}{*}{13} & cogentin (BENZTROPINE) & haldol (HALOPERIDOL) & 97.39 \\
\hline & epitol (CARBAMAZEPINE) & depakote (DIVALPROEX) & 97.31 \\
\hline & fortamet (METFORMIN) & epitol (CARBAMAZEPINE) & 93.56 \\
\hline & riomet (METFORMIN) & cogentin (BENZTROPINE) & 88.88 \\
\hline & cogentin (BENZTROPINE) & glucophage (METFORMIN) & 83.28 \\
\hline & cogentin (BENZTROPINE) & riomet (METFORMIN) & 83.28 \\
\hline & cogentin (BENZTROPINE) & fortamet (METFORMIN) & 73.56 \\
\hline & depakote (DIVALPROEX) & fortamet (METFORMIN) & 68.44 \\
\hline & zyloprim (ALLOPURINOL) & zegerid (OMEPRAZOLE) & 66.24 \\
\hline & aloprim (ALLOPURINOL) & zegerid (OMEPRAZOLE) & 56.09 \\
\hline \multirow[t]{11}{*}{16} & invega (PALIPERIDONE) & pepcid (FAMOTIDINE) & 96.02 \\
\hline & invega (PALIPERIDONE) & flexeril (CYCLOBENZAPRINE) & 89.85 \\
\hline & invega (PALIPERIDONE) & buspar (BUSPIRONE) & 88.77 \\
\hline & flomax (TAMSULOSIN) & buspar (BUSPIRONE) & 88.09 \\
\hline & invega (PALIPERIDONE) & zonegran (ZONISAMIDE) & 82.97 \\
\hline & buspar (BUSPIRONE) & invega (PALIPERIDONE) & 82.35 \\
\hline & zegerid (OMEPRAZOLE) & buspar (BUSPIRONE) & 78.89 \\
\hline & zegerid (OMEPRAZOLE) & zonegran (ZONISAMIDE) & 76.51 \\
\hline & pepcid (FAMOTIDINE) & invega (PALIPERIDONE) & 75.78 \\
\hline & flexeril (CYCLOBENZAPRINE) & invega (PALIPERIDONE) & 72.87 \\
\hline & flexeril (CYCLOBENZAPRINE) & zegerid (OMEPRAZOLE) & 63.85 \\
\hline \multirow[t]{2}{*}{18} & topamax (TOPIRAMATE) & prozac (FLUOXETINE) & 63.25 \\
\hline & topamax (TOPIRAMATE) & sarafem (FLUOXETINE) & 63.25 \\
\hline \multirow[t]{6}{*}{19} & zofran (ONDANSETRON) & prilosec (OMEPRAZOLE) & 97.78 \\
\hline & carafate (SUCRALFATE) & zofran (ONDANSETRON) & 91.55 \\
\hline & prinivil (LISINOPRIL) & zegerid (OMEPRAZOLE) & 77.52 \\
\hline & carafate (SUCRALFATE) & zegerid (OMEPRAZOLE) & 60.55 \\
\hline & lipitor (ATORVASTATIN) & prilosec (OMEPRAZOLE) & 54.83 \\
\hline & lipitor (ATORVASTATIN) & zegerid (OMEPRAZOLE) & 52.83 \\
\hline
\end{tabular}

Table 4.2: Potential Psychotropic Drug-Drug Interactions from 20 separate sets of medication lists for 20 patients. 


\begin{tabular}{|c|c|c|c|c|c|}
\hline Patient & Drug A & Drug B & Confidence & Most Common ADRs Reported & Cases \\
\hline \multirow{3}{*}{5} & zocor & prozac & 84.23 & nausea, fatigue & 7 \\
\hline & zOcor & vistaril & 81.23 & $\begin{array}{l}\text { type } 2 \text { diabetes } \\
\text { caemia, diabetic } \\
\text { caeuropathy, bypergly- } \\
\text { cancer metastatic }\end{array}$ & 2 \\
\hline & zocor & namenda & 64.62 & pulmonary oedema, lethargy & 2 \\
\hline \multirow{5}{*}{8} & tricor & klonopin & 85.21 & vomiting, nausea, dizziness & 4 \\
\hline & tricor & glucophage & 81.69 & coronary artery disease & 6 \\
\hline & plavix & fortamet & 58.16 & muscle spasms & 1 \\
\hline & plavix & tricor & 55.63 & diarrhoea & 9 \\
\hline & zyprexa & plavix & 50.00 & $\begin{array}{l}\text { vomiting, fall, extrapyramidal disorder, } \\
\text { dyspnoea, diarrhoea, chest pain, asthe- } \\
\text { nia }\end{array}$ & 3 \\
\hline \multirow[b]{3}{*}{11} & cogentin & glucophage & 83.28 & $\begin{array}{l}\text { leukocytosis, hypoglycaemia, diabetic } \\
\text { neuropathy }\end{array}$ & 2 \\
\hline & klonopin & vistaril & 64.51 & panic attack & 4 \\
\hline & vistaril & glucophage & 60.31 & $\begin{array}{l}\text { tremor, stress, sleep apnoea syn- } \\
\text { drome,peripheral coldness, memory im- } \\
\text { pairment, incorrect dose administered, } \\
\text { hypoaesthesia, hyperhidrosis, feeling } \\
\text { abnormal, drug tolerance, chills }\end{array}$ & 1 \\
\hline \multirow[t]{2}{*}{13} & cogentin & haldol & 97.39 & $\begin{array}{l}\text { schizophrenia, renal failure acute, poi- } \\
\text { soning, neutrophil count decreased, } \\
\text { neuroleptic malignant syndrome, men- } \\
\text { tal disorder, inappropriate schedule of } \\
\text { drug administration, dysphagia, drug } \\
\text { abuse, constipation, bradycardia, ag- } \\
\text { gression }\end{array}$ & 2 \\
\hline & cogentin & glucophage & 83.28 & $\begin{array}{l}\text { leukocytosis, hypoglycaemia, diabetic } \\
\text { neuropathy }\end{array}$ & 2 \\
\hline \multirow[t]{2}{*}{16} & flomax & buspar & 88.09 & $\begin{array}{l}\text { type } 2 \text { diabetes mellitus, pneumonia, } \\
\text { hypoglycaemia, diabetes mellitus, chest } \\
\text { pain, cardiac disorder, angina pectoris }\end{array}$ & 1 \\
\hline & flexeril & zegerid & 63.85 & fibromyalgia & 1 \\
\hline \multirow[b]{2}{*}{18} & topamax & prozac & 63.25 & drug ineffective & 6 \\
\hline & topamax & sarafem & 63.25 & $\begin{array}{l}\text { vision blurred, speech disorder, paraes- } \\
\text { thesia, muscular weakness, motor } \\
\text { dysfunction, hypoaesthesia, headache, } \\
\text { dysarthria, dizziness, confusional state, } \\
\text { cerebrovascular accident, aphasia, aba- } \\
\text { sia }\end{array}$ & 2 \\
\hline \multirow{4}{*}{19} & zofran & prilosec & 97.78 & pain & 45 \\
\hline & carafate & zofran & 91.55 & dysphagia, anaemia & 7 \\
\hline & lipitor & prilosec & 54.83 & pain & 46 \\
\hline & lipitor & zegerid & 52.83 & arthralgia & 1 \\
\hline
\end{tabular}

Table 4.3: Potential Psychotropic DDI ADRs for drug-pairs listed in Table 4.2. The table also shows the most common side effects and number of cases reported in the 2010 Q4 AERS data set. 


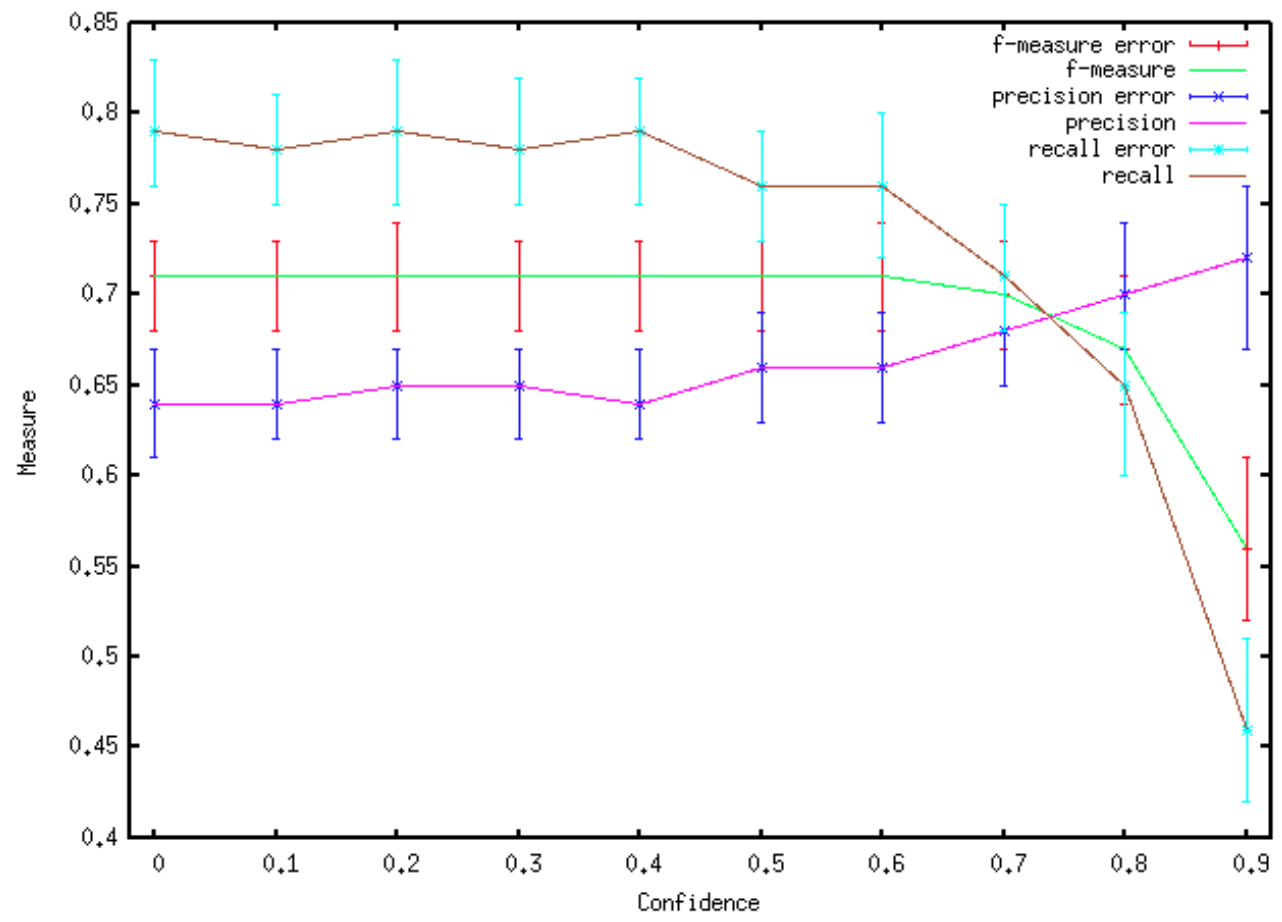

Figure 4.3: Evaluation of SVM classifier on 100 positive and 100 unlabeled instances.

\subsubsection{Statistical Analysis}

To evaluate the credibility of the dataset and establish a more concrete validation for our classification results, we performed a one-way Analysis of Variance on the drug features in order to comprehend the correlation or dissimilarity between known DDIs $\left(\lambda_{k}\right)$, potential DDIs $\left(\lambda_{p}\right)$ and unknown DDIs $\left(\lambda_{u}\right)$. The null hypothesis here indicates that the drug attributes used in this work have no influence when distinguishing between known, predicted and unknown DDIs. If an attribute's F-test score is greater than the critical value, then we reject the null hypothesis and accept the fact that known and potential DDIs are indeed distinguishable from the unlabeled set, since we initially assumed them (known and potential DDIs) to possess similar characteristics. Furthermore, rejecting the null hypothesis enables us to identify the most influential attributes.

For a given attribute $x$, it's F-test score was computed as follows: For each of the three categories: $\lambda_{k}, \lambda_{p}, \lambda_{u}$, randomly select $n$ samples of attribute $x_{i}$, where $x_{1}=\lambda_{k}, x_{2}=\lambda_{p}$ and $x_{3}=\lambda_{u}$. The null hypothesis, denoted $H_{0}$, for the overall F-test is that all three categories produce the same response on average. Suppose we let $x=$ attribute 20 (ppi) 


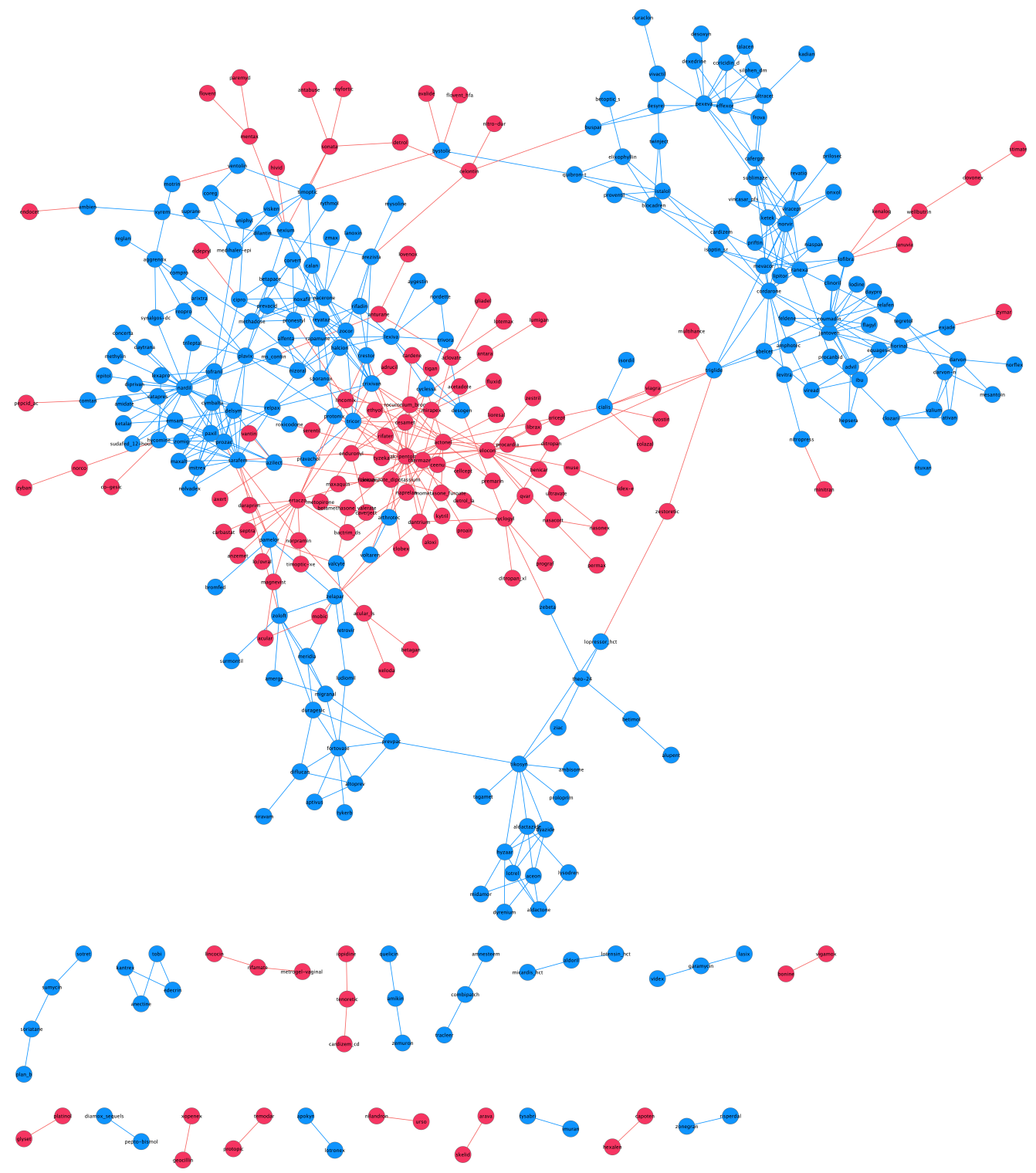

Figure 4.4: Network showing drugs involved in known (blue nodes, 397 pairs) and predicted (red nodes, 181 pairs) drug-drug interactions. Blue edges indicate a connection between two nodes known to interact prior to experiments while red edges represent probable connections predicted by our system. 


\begin{tabular}{|c|c|c|}
\hline Drug A & Drug B & Confidence \\
\hline provigil & antabuse & $98.67 \%$ \\
\hline aricept & antabuse & $98.66 \%$ \\
\hline zoloft & prozac & $98.18 \%$ \\
\hline zoloft & trileptal & $97.14 \%$ \\
\hline antabuse & restoril & $96.80 \%$ \\
\hline celexa & antabuse & $96.72 \%$ \\
\hline ambien & librium & $96.32 \%$ \\
\hline wellbutrin & provigil & $95.54 \%$ \\
\hline provigil & klonopin & $95.16 \%$ \\
\hline ambien & antabuse & $94.92 \%$ \\
\hline vistaril & antabuse & $93.86 \%$ \\
\hline zoloft & provigil & $93.01 \%$ \\
\hline klonopin & librium & $92.73 \%$ \\
\hline paxil & depakote & $92.29 \%$ \\
\hline paxil & aricept & $91.69 \%$ \\
\hline abilify & ambien & $91.60 \%$ \\
\hline paxil & prozac & $90.44 \%$ \\
\hline valium & vistaril & $90.06 \%$ \\
\hline haldol & buspar & $89.92 \%$ \\
\hline antabuse & ambien & $89.77 \%$ \\
\hline librium & ambien & $89.03 \%$ \\
\hline trileptal & namenda & $88.69 \%$ \\
\hline provigil & cymbalta & $88.34 \%$ \\
\hline klonopin & antabuse & $87.57 \%$ \\
\hline strattera & ritalin & $87.50 \%$ \\
\hline wellbutrin & vistaril & $87.23 \%$ \\
\hline aricept & paxil & $87.12 \%$ \\
\hline concerta & wellbutrin & $85.69 \%$ \\
\hline ambien & concerta & $84.72 \%$ \\
\hline paxil & strattera & $84.54 \%$ \\
\hline valium & antabuse & $84.29 \%$ \\
\hline prozac & klonopin & $82.95 \%$ \\
\hline prozac & keppra & $81.30 \%$ \\
\hline concerta & cymbalta & $81.05 \%$ \\
\hline cymbalta & ritalin & $81.05 \%$ \\
\hline zoloft & celexa & $80.65 \%$ \\
\hline restoril & ambien & $79.61 \%$ \\
\hline concerta & zoloft & $79.27 \%$ \\
\hline wellbutrin & ritalin & $78.76 \%$ \\
\hline buspar & abilify & $78.76 \%$ \\
\hline antabuse & keppra & $78.55 \%$ \\
\hline paxil & navane & $78.52 \%$ \\
\hline navane & buspar & $78.31 \%$ \\
\hline paxil & wellbutrin & $78.29 \%$ \\
\hline antabuse & klonopin & $75.85 \%$ \\
\hline
\end{tabular}

\begin{tabular}{|c|c|c|}
\hline Drug A & Drug B & Confidence \\
\hline zoloft & ritalin & $75.70 \%$ \\
\hline zoloft & concerta & $75.70 \%$ \\
\hline paxil & celexa & $75.33 \%$ \\
\hline klonopin & lunesta & $75.33 \%$ \\
\hline librium & paxil & $74.81 \%$ \\
\hline paxil & abilify & $73.66 \%$ \\
\hline vistaril & ritalin & $71.67 \%$ \\
\hline prozac & paxil & $71.52 \%$ \\
\hline cymbalta & haldol & $69.41 \%$ \\
\hline antabuse & valium & $68.85 \%$ \\
\hline keppra & provigil & $65.00 \%$ \\
\hline keppra & wellbutrin & $65.00 \%$ \\
\hline topamax & librium & $64.95 \%$ \\
\hline strattera & keppra & $64.81 \%$ \\
\hline topamax & antabuse & $64.64 \%$ \\
\hline klonopin & vistaril & $64.51 \%$ \\
\hline paxil & vistaril & $64.51 \%$ \\
\hline topamax & prozac & $63.25 \%$ \\
\hline keppra & vistaril & $62.54 \%$ \\
\hline haldol & abilify & $61.67 \%$ \\
\hline keppra & buspar & $61.31 \%$ \\
\hline neurontin & prozac & $61.22 \%$ \\
\hline wellbutrin & paxil & $60.91 \%$ \\
\hline lexapro & ritalin & $60.56 \%$ \\
\hline strattera & paxil & $58.79 \%$ \\
\hline restoril & klonopin & $58.79 \%$ \\
\hline librium & valium & $57.99 \%$ \\
\hline paxil & ritalin & $57.90 \%$ \\
\hline paxil & concerta & $57.90 \%$ \\
\hline buspar & navane & $57.35 \%$ \\
\hline serzone & aricept & $56.75 \%$ \\
\hline provigil & keppra & $56.61 \%$ \\
\hline wellbutrin & keppra & $56.61 \%$ \\
\hline celexa & keppra & $56.22 \%$ \\
\hline aricept & neurontin & $55.77 \%$ \\
\hline concerta & ritalin & $55.44 \%$ \\
\hline keppra & aricept & $55.33 \%$ \\
\hline paxil & haldol & $55.07 \%$ \\
\hline buspar & keppra & $54.56 \%$ \\
\hline effexor & celexa & $53.89 \%$ \\
\hline trileptal & topamax & $52.74 \%$ \\
\hline serzone & celexa & $52.05 \%$ \\
\hline concerta & paxil & $51.32 \%$ \\
\hline paxil & zoloft & $51.00 \%$ \\
\hline cymbalta & prolixin & $50.88 \%$ \\
\hline
\end{tabular}

Table 4.4: DDI for Psychotropic medications found in the unlabeled drug-pair dataset 
and $n=41$, such that $x_{i}=\left\{x_{i 1}, x_{i 2}, \ldots, x_{i 41}\right\}$ represents the 41 randomly chosen values for $x_{i}$, then the F-ratio can be obtained by:

Step 1: Calculating the mean within each group:

$$
\overline{Y_{i}}=\frac{1}{n} \sum_{j=1}^{n} Y_{i j}, \quad i=1,2, . ., g
$$

where $g$ is the total number of groups

Step 2: Calculating the overall mean

$$
\bar{Y}=\frac{\sum_{i} \overline{Y_{i}}}{g}, \quad g=3
$$

Step 3: Calculate "between-group" sum of squares:

$$
S_{B}=n\left(\bar{Y}_{1}-\bar{Y}\right)^{2}+n\left(\bar{Y}_{2}-\bar{Y}\right)^{2}+n\left(\bar{Y}_{3}-\bar{Y}\right)^{2}
$$

The between-group degrees of freedom is one less than the number of groups

$$
f_{b}=a-1
$$

so the between-group mean square value is

$$
M S_{B}=\frac{S_{B}}{f_{b}}
$$

Step 4: Calculate the within-group sum of squares

$$
S_{W}=\sum_{i=1}^{g} \sum_{j=1}^{n}\left(x_{i j}-\bar{Y}_{i}\right)^{2}
$$

The within-group degrees of freedom is

$$
f_{w}=g(n-1)
$$


Thus, the within-group mean square value is

$$
M S_{W}=\frac{S_{W}}{f_{W}}
$$

Step 5: The F-ratio is

$$
F=\frac{M S_{B}}{M S_{W}}
$$

Applying Equation 4.1 on the group elements tabulated in Figure 4.5, we obtain a mean of $0.03,0.04$ and 0.14 for $\lambda_{k}, \lambda_{p}$ and $\lambda_{u}$ respectively, giving an overall mean of 0.07 applying Equation 4.2. Computing the between-group sum of squares gives 0.30, and with a between-group degrees of freedom of $2, M S_{B}$ gives 0.15 . Following Step 4, we computed a within-group sum of squares of 2.66 , and with $f_{b}=120, M S_{W}$ gave 0.02 , producing a final F-ratio of 7.5 .

Since for each attribute, values were selected at random, this process was repeated 100 times and the average was selected as the final F-ratio. Data samples from this process have been listed in Table 4.6 to highlight the difference in the distribution of $\mathrm{K}$ and $\mathrm{P}$ from that of $\mathrm{U}$. The F-ratio for the top 15 attributes selected during the feature subset selection process is presented in 4.1, showing the average F-ratio for Protein-Protein Interactions as a feature as 7.5. The critical value is the number that the test statistic must exceed to reject the null hypothesis. In this case, $F_{\text {crit }}(2,120)=3.07$ at a significance level of 0.05 . Since $F=7.5>3.07$, the results are statistically significant and therefore we can safely reject the null hypothesis. The p-value for this test is 0.000180 . From the table it can be noted that Human Protein Targets serve as the best determinant for distinguishing known and potential drug-drug interactions from unknown ones. To demonstrate this, we plot the distribution of binary protein-protein interactions ( $p p_{-} b$ - attribute index 20$)$ against the distribution of structural formula similarity (sfmsim - attribute index 22), and present the results in Figure 4.6. 


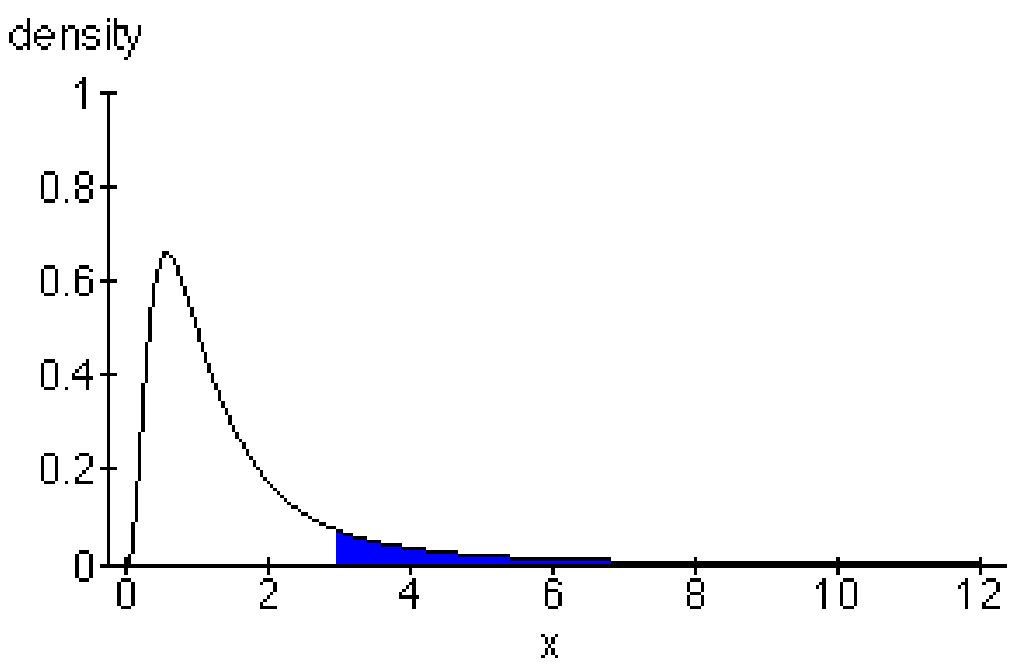

Figure 4.5: F-Distribution showing critical value $\mathrm{F}(2,120)=3.07$, which is the starting point of the blue shaded region indicating the area within which the null hypothesis is rejected.

\begin{tabular}{|l|l|l|l|l|l|l|l|l|l|l|l|l|l|l|l|l|l|l|l|l|l|l|l|l|l|}
\hline K & 0.0 & 0.0 & 0.0 & 0.0 & 0.1 & 0.0 & 0.0 & 0.0 & 0.0 & 0.3 & 0.0 & 0.0 & 0.0 & 0.0 & 0.0 & 0.2 & 0.0 & 0.2 & 0.0 & 0.0 & 0.2 & 0.4 & 0.0 & 0.0 & 0.0 \\
\hline
\end{tabular}

\begin{tabular}{llllllllllllllllllllllllllllllllll}
\hline$P$ & 0.0 & 0.1 & 0.1 & 0.2 & 0.0 & 0.0 & 0.0 & 0.2 & 0.1 & 0.0 & 0.0 & 0.0 & 0.1 & 0.2 & 0.2 & 0.0 & 0.0 & 0.0 & 0.0 & 0.0 & 0.0 & 0.0 & 0.0 & 0.1 & 0.1 \\
\hline
\end{tabular}

\begin{tabular}{lll|l|l|l|l|l|l|l|l|l|l|l|l|l|l|l|l|l|l|l|l|l|l|l|l|}
\hline $\mathrm{U}$ & 0.3 & 0.4 & 0.3 & 0.0 & 0.1 & 1.0 & 0.7 & 0.3 & 0.3 & 0.1 & 0.5 & 0.1 & 0.3 & 0.0 & 0.0 & 0.0 & 0.2 & 0.3 & 0.7 & 0.1 & 0.0 & 0.0 & 0.2 & 0.0 & 0.0 \\
\hline
\end{tabular}

Table 4.5: Forty-one randomly selected attribute-values for attribute ppi_b (index 20). K, $\mathrm{P}$, U represent known DDIs, predicted DDIs and unknown DDIs respectively. Columns containing no information (0.0) across all three rows have been compressed 


\begin{tabular}{|r|c|c|c|c|c|c|c|c|c|c|c|c|}
\multicolumn{1}{c|}{} & \multicolumn{19}{c|}{ K } & \multicolumn{1}{c|}{ P } & \multicolumn{4}{c|}{ U } \\
\hline Run & mean & std & min & max & mean & std & min & max & mean & std & min & max \\
\hline 1 & 0.02 & 0.05 & 0.00 & 0.17 & 0.02 & 0.08 & 0.00 & 0.38 & 0.15 & 0.23 & 0.00 & 0.75 \\
\hline 2 & 0.03 & 0.10 & 0.00 & 0.50 & 0.05 & 0.10 & 0.00 & 0.50 & 0.10 & 0.15 & 0.00 & 0.67 \\
\hline 3 & 0.02 & 0.06 & 0.00 & 0.25 & 0.02 & 0.09 & 0.00 & 0.50 & 0.13 & 0.17 & 0.00 & 0.50 \\
\hline 4 & 0.02 & 0.09 & 0.00 & 0.50 & 0.02 & 0.04 & 0.00 & 0.17 & 0.11 & 0.14 & 0.00 & 0.50 \\
\hline 5 & 0.02 & 0.09 & 0.00 & 0.50 & 0.03 & 0.07 & 0.00 & 0.38 & 0.13 & 0.19 & 0.00 & 0.75 \\
\hline 6 & 0.01 & 0.03 & 0.00 & 0.12 & 0.05 & 0.14 & 0.00 & 0.67 & 0.13 & 0.24 & 0.00 & 1.00 \\
\hline 7 & 0.02 & 0.05 & 0.00 & 0.17 & 0.02 & 0.07 & 0.00 & 0.36 & 0.15 & 0.24 & 0.00 & 1.00 \\
\hline 8 & 0.02 & 0.08 & 0.00 & 0.40 & 0.02 & 0.06 & 0.00 & 0.33 & 0.20 & 0.21 & 0.00 & 1.00 \\
\hline 9 & 0.01 & 0.05 & 0.00 & 0.28 & 0.04 & 0.12 & 0.00 & 0.56 & 0.10 & 0.15 & 0.00 & 0.50 \\
\hline 10 & 0.04 & 0.13 & 0.00 & 0.67 & 0.04 & 0.07 & 0.00 & 0.33 & 0.14 & 0.25 & 0.00 & 1.00 \\
\hline 11 & 0.03 & 0.08 & 0.00 & 0.33 & 0.02 & 0.05 & 0.00 & 0.20 & 0.12 & 0.21 & 0.00 & 1.00 \\
\hline 12 & 0.05 & 0.13 & 0.00 & 0.50 & 0.03 & 0.09 & 0.00 & 0.50 & 0.12 & 0.21 & 0.00 & 1.00 \\
\hline 13 & 0.02 & 0.08 & 0.00 & 0.50 & 0.03 & 0.09 & 0.00 & 0.50 & 0.12 & 0.14 & 0.00 & 0.33 \\
\hline 14 & 0.04 & 0.12 & 0.00 & 0.64 & 0.02 & 0.06 & 0.00 & 0.25 & 0.17 & 0.18 & 0.00 & 0.67 \\
\hline 15 & 0.06 & 0.15 & 0.00 & 0.67 & 0.01 & 0.03 & 0.00 & 0.12 & 0.16 & 0.23 & 0.00 & 1.00 \\
\hline 16 & 0.03 & 0.09 & 0.00 & 0.50 & 0.01 & 0.04 & 0.00 & 0.17 & 0.17 & 0.24 & 0.00 & 1.00 \\
\hline 17 & 0.04 & 0.08 & 0.00 & 0.33 & 0.04 & 0.09 & 0.00 & 0.50 & 0.14 & 0.19 & 0.00 & 0.50 \\
\hline 18 & 0.04 & 0.10 & 0.00 & 0.50 & 0.03 & 0.08 & 0.00 & 0.27 & 0.13 & 0.16 & 0.00 & 0.50 \\
\hline 19 & 0.03 & 0.12 & 0.00 & 0.67 & 0.08 & 0.15 & 0.00 & 0.67 & 0.14 & 0.17 & 0.00 & 0.67 \\
\hline 20 & 0.02 & 0.06 & 0.00 & 0.28 & 0.03 & 0.07 & 0.00 & 0.33 & 0.13 & 0.24 & 0.00 & 1.00 \\
\hline 21 & 0.03 & 0.09 & 0.00 & 0.50 & 0.02 & 0.04 & 0.00 & 0.17 & 0.18 & 0.20 & 0.00 & 0.67 \\
\hline Avg & 0.03 & 0.09 & 0.00 & 0.43 & 0.03 & 0.08 & 0.00 & 0.37 & 0.14 & 0.20 & 0.00 & 0.76 \\
\hline
\end{tabular}

Table 4.6: Twenty-one samples of data obtained from the process described in Table 4.5. The mean, standard deviation (std), minimum and maximum values for $\mathrm{K}, \mathrm{P}$ and $\mathrm{U}$ has been computed for each iteration. From the table, it can be observed that the $\mathrm{P}$ and $\mathrm{K}$ have similar distributions, both of which are different from $\mathrm{U}$. 


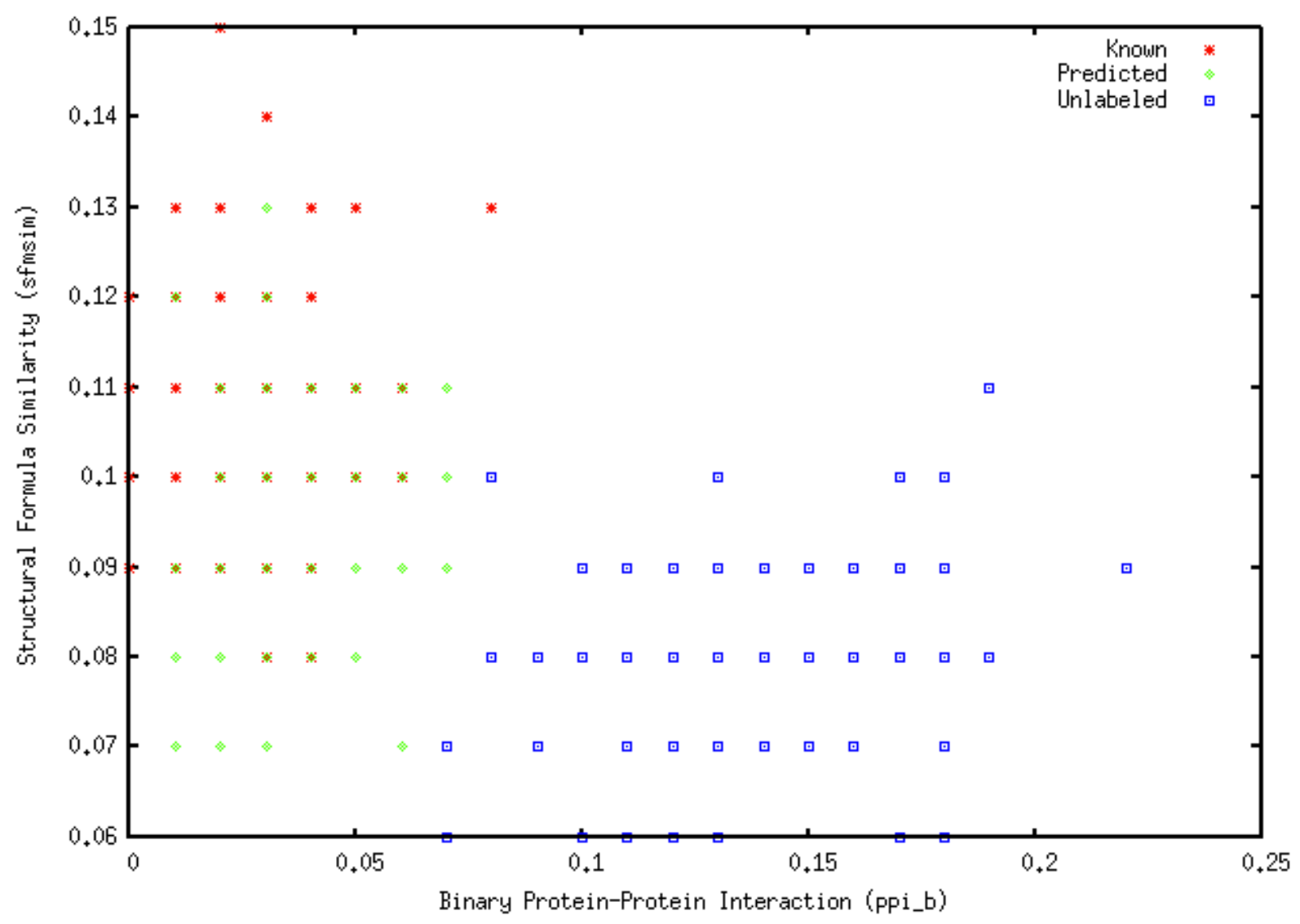

Figure 4.6: Distribution of Known, Predicted and Unlabeled instances based on mean values of attributes ppi_b (binary protein-protein interaction) and sfmsim (structural formula similarity) for 41 randomly selected drug-pairs, and repeated 100 times. Therefore each class (i.e. K, P and U) plotted consists of exactly 100 points, some of which overlap each other. The plot clearly shows a separation of clusters of $\mathrm{K}$ and $\mathrm{P}$ from $\mathrm{U}$. 


\section{Chapter 5}

\section{Conclusion and Future Work}

\subsection{Conclusion}

By combining drug attributes in a pharmacological space and employing Machine Learning techniques to identify unique patterns in this space we have been able to establish a novel framework for automating the prediction of potential drug-drug interactions for clinical drugs in a matter of minutes, completely bypassing traditional in vivo and/or in vitro methods which usually take a significant amount of time to complete.

In this work, we have presented a novel approach and framework for automating the detection of drug-drug interactions among clinically approved drugs. This was accomplished by exploiting a number of drug attributes and their similarities, combining them in a pharmacological space and employing machine learning techniques to identify patterns of interest within this multidimensional space.

Furthermore, in this framework, we have introduced and addressed a very critical, yet interesting problem of inverse feature vectors when constructing datasets based on drugpairs. A problem which becomes very sophisticated due to the generation of highly complex local patterns as more drugs are combined to create a single feature vector. We have provided a modified version of the PU-learning algorithm which addresses the case for two drugs by choosing the class label assigned to a feature vector, or its inverse, with the 
maximum probability of membership.

\subsection{Future Work}

The feature space could be expanded to include a number of other drug attributes such as polarity and blackbox information.

Our initial intention was to use the Smith-Waterman algorithm for protein sequence alignment but due to its demanding nature in terms of time and memory resources especially when processing millions of entries we had to consider other alternatives, hence the use of BLAST 2 in this work. Although BLAST is not guaranteed to find optimal alignments, it is much more practical and efficient. Another well known algorithm is FastA (Fast Alignment) [49], an extension of FastP (for protein alignment) and FastN (for nucleotide) alignment.

Furthermore, implementation of an automatic validation mechanism for false positive DDIs via web content mining could prove very useful in this context. This can be achieved by scraping social media including micro-blogging sites such as Twitter, public forums such as Momcafe, news websites and popular social networks such as Facebook. This would warrant the identification of real world cases where people may have encountered adverse pharmacological effects attributable to drug-drug interactions and posted their experiences on the Internet.

Moreover, an optimal solution to the inverse feature vectors problem so that only one feature vector is required for any given drug-pair will cut the computation time in half, all things being equal. Extending feature vectors to support more than two drugs may require new data structures to be developed in order to support the complex vectors they produce.

Finally, automating the process of collecting drug-attribute information from the various data sources such as FDA's SPL database, KEGG/LIGAND database and DrugBank in order to facilitate rapid construction of feature vectors for new drugs and render the detection of DDI using this framework even more practical. 


\subsection{Broader Application Domains}

The methodology presented and employed in this research can be extended to a number of other domains. For instance, in the case of a car manufacturing company such as Toyota, this could provide a solution to the mechanical malfunction that led to the 2009-2011 vehicle recalls [50]. Here, the positive set would comprise data collected from faulty vehicles while data from all other vehicles would make up the unlabeled set. Classifiers can then be trained to predict potentially faulty vehicles amongst the unlabeled set.

Additionally, the system could be modified to identify websites of common interest. In the simplest sense, this can be accomplished by generating a list of websites, pairing the entries, and then creating feature vectors based on certain attributes pertaining to each website's content such as word-count, presence of certain HTML tags and Cascading Style Sheet (CSS) properties, number of hyperlinks, number and type of multimedia files embedded and so on. By identifying the positive set, that is, website-pairs that are known to be similar such as Cable News Network (CNN) and FOX News (both broadcast breaking news and other information regarding business, weather, entertainment, etc), classifiers can be trained to expand this positive set by identifying potential website-pairs among the unlabeled set that may share some degree of similarity. 


\section{Appendix A}

\section{Tools}

\section{A.1 Python}

All pre- and post-processing was accomplished with Python 2.6.1 (r261:67515, Jun 24 2010, 21:47:49).

\section{A.2 GNUPLOT}

GNUPLOT is the primary graphing utility used in this project. Specifically GNUPLOT Version 4.4 patchlevel 3, which was last modified on March 2011.

\section{A.3 Cytoscape}

All network visualizations accompanying this project were designed in Cytoscape 2.8.1 an open source software platform for visualizing complex networks and integrating these networks with any type of attribute data [51]. 


\section{Bibliography}

[1] D. Gusfield, Algorithms on Strings, Trees, and Sequences: Computer Science and Computational Biology. Cambridge University Press, 1997.

[2] P. Vonbach, A. Dubied, K. Stephen, and J. H. Beer, "Evaluation of frequently used drug interaction screening programs.," Pharm World Sci, vol. 30, no. 4, pp. 367-74, 2008.

[3] U.S. Food And Drug Administration (FDA), "FDA Structured Product Labeling." DailyMed http://dailymed. nlm.nih.gov/dailymed/about. $c f m ? C F I D=34386183 \& C F T O K E N=$ 93c73848d4b2d351-812E3B58-B063-DCED-2E081B641B586A14\&j sessionid= ca307d1a7961517f6570.

[4] K. Baxter (ed), Stockley's Drug Interactions 9 [CD-ROM] London, 2010.

[5] DrugBank, "DrugCard Version 3.0." http://drugbank.ca/system/downloads/2.5/ drugcards.zip (last accessed December 3, 2010), January 2009.

[6] FD\&C Act, sec. 201(g)(1), "Federal food, drug, and cosmetic act (fd\&c act)." http://www.fda.gov/RegulatoryInformation/ Legislation/FederalFoodDrugandCosmeticActFDCAct/ FDCActChaptersIandIIShortTitleandDefinitions/ucm086297.htm, July 2009.

[7] World Health Organization, "International drug monitoring: The role of national centres," WHO Technical Report Series, no. 498, 1972. 
[8] J. Lazarou, B. H. Pomeranz, and P. N. Corey, "Incidence of adverse drug reactions in hospitalized patients: a meta-analysis of prospective studies," JAMA, vol. 279, no. 1200-5, 1998.

[9] S.-F. Lin, K.-T. Xiao, Y.-T. Huang, and V.-W. Soo, "A tool for finding possible explanation for adverse drug reactions through drug and drug target interactions," in BioMedical Engineering and Informatics, 2008. BMEI 2008. International Conference on, vol. 1, pp. $580-584$, May 2008.

[10] T. D. Bjornsson, J. T. Callaghan, H. J. Einolf, V. Fischer, L. Gan, S. Grimm, J. Kao, S. P. King, G. Miwa, L. Ni, G. Kumar, J. McLeod, R. S. Obach, S. Roberts, A. Roe, A. Shah, F. Snikeris, J. T. Sullivan, D. Tweedie, J. M. Vega, J. Walsh, and S. A. Wrighton, "The conduct of in vitro and in vivo drug-drug interaction studies: A pharmaceutical research and manufacturers of america (phrma) perspective," Drug Metabolism and Disposition, vol. 31, pp. 815-832, July 2003.

[11] K. A. Phillips, D. L. Veenstra, E. Oren, J. K. Lee, and W. Sadee, "Potential role of pharmacogenomics in reducing adverse drug reactions: A systematic review," JAMA, vol. 286, November 142001.

[12] T. Arikuma, S. Yoshikawa, K. Watanabe, K. Matsumura, and A. Konagaya, "Ontologydriven hypothetic assertion (oha) for drug interactionprediction," in Computer and Computational Sciences, 200\%. IMSCCS 200\%. Second International Multi-Symposiums on, pp. $1-8$, aug. 2007.

[13] D. K. Badyal and A. P. Dadhich, "Cytochrome p450 and drug interactions," Indian Journal of Pharmacology, vol. 33, pp. 248-259, October 32001.

[14] K. E. Ferslew, A. N. Hagardorn, G. C. Harlan, and W. F. McCormick, "A fatal drug interaction between clozapine and fluoxetine," Journal of Forensic Science, vol. 43, pp. 1082-5, September 1998.

[15] E. Spina, A. Avenoso, G. Facciolà, M. Fabrazzo, P. Monteleone, M. Maj, E. Perucca, and A. P. Caputi, "Effect of fluoxetine on the plasma concentrations of clozapine and its 58 
major metabolites in patients with schizophrenia.," International Clinical Psychopharmacology, vol. 13, no. 3, pp. 141-145, 1998.

[16] B. P. Monahan, C. L. Ferguson, E. S. Killeavy, B. Lloyd, J. Troy, and L. R. Cantilena, "Torsades de pointes occurring in association with terfenadine use," JAMA, vol. 264, pp. 2788-2790, 1990.

[17] N. R. Crowther, A. M. Holbrook, R. Kenwright, and M. Kenwright, "Drug interactions among commonly used medications," CME, vol. 43, November 1997.

[18] M. A. Yildrim, K. L. Goh, M. E. Cusick, A. L. Barabasi, and M. Vidal, "Drug-target network," Computational Biology, vol. 25, October 2007.

[19] A. Ma'ayan, S. L. Jenkins, J. Goldfarb, and R. Lyengar, "Network analysis of FDA approved drugs and their targets," NIH, vol. 74, pp. 27-32, April 2007.

[20] J. Scheiber, J. L. Jenkins, S. C. K. Sukuru, A. Bender, D. Mikhailov, M. Milik, K. Azzaoui, S. Whitebread, J. Hamon, L. Urban, M. Glick, and J. W. Davies, "Mapping adverse drug reactions in chemical space," Journal of Medicinal Chemistry, vol. 52, no. 9, pp. 3103-3107, 2009.

[21] Elsevier, "Pharmapendium." https://www.pharmapendium.com/, 2008.

[22] M. Campillos, M. Kuhn, A.-C. Gavin, L. J. Jensen, and P. Bork, "Drug target identification using side-effect similarity," Science, vol. 321, pp. 263-266, 2008.

[23] S. Zhao and S. Li, "Network-based relating pharmacological and genomic spaces for drug target identification," PLoS ONE, vol. 5, July 2010.

[24] Z. Spiro, I. A. Kovacs, and P. Csermely, "Drug-therapy networks and the prediction of novel drug targets," Journal of Biology, July 2008.

[25] FDA U.S. Food and Drug Administration, "The adverse event reporting system (AERS): Latest quarterly data files." http://www.fda.gov/ downloads/Drugs/GuidanceComplianceRegulatoryInformation/Surveillance/ AdverseDrugEffects/UCM251745.zip, December 2010. 
[26] J. Strandell and S. Wahlin, "Pharmacodynamic and pharmacokinetic drug interactions reported to vigibase, the who global individual case safety report database.," Eur J Clin Pharmacol, 2011.

[27] WHO Collaborating Center for Drug Statistics Methodology (WHOCC), "ATC Structure and Principles." http://www.whocc.no/atc/structure_and_principles/, 2011. [Online; accessed 03-Mar-2011].

[28] R. Hanzlik, Y. Koen, B. Theertham, Y. Dong, and J. Fang, "The reactive metabolite target protein database (tpdb) - a web-accessible resource," BMC Bioinformatics, vol. 8, no. 1, p. 95, 2007.

[29] E. M. Phizicky and S. Fields, "Protein-protein interactions: Methods for detection and analysis," Microbiological Reviews, vol. 59, pp. 94-123, March 1995.

[30] C. Stark, B. J. Breitkreutz, T. Reguly, L. Boucher, A. Breikreutz, M. Tyers, and all contributing authors, "A general repository for interaction datasets.," Nucleic Acids Res, vol. 34, Jan 12010.

[31] S. F. Altschul, W. Gish, W. Miller, E. W. Myers, and D. J. Lipman, "Basic local alignment search tool," Journal of Molecular Biology, vol. 215, pp. 403-10, Oct 51990.

[32] S. F. Altschul, T. L. Madden, A. A. Schaffer, J. Zhang, W. Miller, and D. J. Lipman, "Gapped blast and psi-blast: a new generation of protein database search programs," Nucleic Acids Res, vol. 25, pp. 3389-402, Sep 11997.

[33] T. F. Smith and M. S. Waterman, "Identification of common molecular subsequences," Journal of Molecular Biology, vol. 147, pp. 195-197, 1981.

[34] S. B. Needleman and C. D. Wunsch, "A general method applicable to the search for similarities in the amino acid sequence of two proteins," Journal of Molecular Biology, vol. 48, no. 3, pp. $443-453,1970$.

[35] T. A. Tatusova and T. L. Madden, "Blast 2 sequences, a new tool for comparing protein and nucleotide sequences," FEMS Microbiology Letters, vol. 174, no. 2, pp. 247-250, 1999. 
[36] M. Kanehisa, S. Groto, M. Furumichi, M. Tanabe, and M. Hirakawa, "Kegg for representation and analysis of molecular networks involving diseases and drugs.," Nuclieic Acids Res, vol. 38, pp. D355-D360, 2010.

[37] M. Kanehisa, S. Goto, M. Hattori, K. F. Aoki-Kinoshita, M. Itoh, S. Kawashima, T. Katayama, M. Araki, and M. Hirakawa, "From genomics to chemical genomics: new developments in kegg," Nucleic Acids Res, vol. 34, pp. D354-357, 2006.

[38] M. Kanehisa and S. Goto, "Kegg: Kyoto encyclopedia of genesa and genomes," Nuclieic Acids Res, vol. 28, pp. 27-30, 2000.

[39] B. Liu, W. S. Lee, P. S. Yu, and X. Li, "Partially supervised classification of text documents," 19th Int'l Conf. Machine Learning, 2002.

[40] H. Yu, J. Han, and K. C.-C. Chang, "Pebl: positive example based learning for web page classification using svm.," in KDD'02, pp. 239-248, 2002.

[41] C. Elkan and K. Noto, "Learning classifiers from only positive and unlabeled data," in Proceeding of the 14th ACM SIGKDD international conference on Knowledge discovery and data mining, KDD '08, (New York, NY, USA), pp. 213-220, ACM, 2008.

[42] W. S. Lee and B. Liu, "Learning with positive and unlabeled examples using weighted logistic regression," Twentieth International Conference on Machine Learning, August 21-24 2003.

[43] P. Perner, ed., Machine Learning and Data Mining in Pattern Recognition, 5th International Conference, MLDM 2007, Leipzig, Germany, July 18-20, 2007, Proceedings, vol. 4571 of Lecture Notes in Computer Science, Springer, 2007.

[44] A. Blum and T. Mitchel, "Combining labeled and unlabeled data with co-training," ACM, vol. 1, no. 58113-057, 1998.

[45] B. Zhang and W. Zuo, "Tri-training based learning from positive and unlabeled data," International Symposiums on Information Processing, 2008. 
[46] N. Bhardwaj, M. Gerstein, and H. Lu, "Genome-wide sequence-based prediction of peripheral proteins using a novel semi-supervised learning technique," BMC Bioinformatics, vol. 11, no. Suppl 1, p. S6, 2010.

[47] Z.-H. Zhou and M. Li, "Tri-training: Exploiting unlabeled data using three classifiers," IEEE Trans. on Knowl. and Data Eng., vol. 17, pp. 1529-1541, November 2005.

[48] C.-C. Chang and C.-J. Lin, LIBSVM: a library for support vector machines, 2001. Software available at http://www.csie.ntu.edu.tw/ cjlin/libsvm.

[49] A. M. Griffin and H. G. Griffin, eds., Computer Analysis of Sequence Data Methods in Molecular Biology, vol. 25 of 365-389. 10.1385/0-89603-276-0:365. International Union of Biochemistry and Molecular Biology, 1994.

[50] Toyota Motor, "2009-2011 toyota vehicle recall." http://pressroom.toyota.com/ safety-recall/, June 292011.

[51] M. E. Smoot, K. Ono, J. Ruscheinski, P.-L. Wang, and T. Ideker, "Cytoscape 2.8," Bioinformatics, vol. 27, pp. 431-432, February 2011. 